\section{Risk-Adjusted Linearizations of Dynamic Equilibrium Models}

\author{
Pierlauro Lopez, ${ }^{1}$ David Lopez-Salido ${ }^{2}$ \\ $\&$ Francisco Vazquez-Grande ${ }^{2}$
}

\author{
December 2018, WP 702
}

\begin{abstract}
We propose a simple risk-adjusted linear approximation to solve a large class of dynamic models with time-varying and non-Gaussian risk. Our approach generalizes lognormal affine approximations commonly used in the macro-finance literature and can be seen as a first-order perturbation around the risky steady state. Therefore, we unify coexisting theories of risk-adjusted linearizations. We provide a formal foundation for approximation methods that remained so far heuristic, and offer explicit formulas for approximate equilibrium objects and conditions for their local existence and uniqueness. Affine approximations are not nested in conventional perturbations of arbitrary order. We apply this technique to models featuring Campbell-Cochrane habits, recursive preferences, and time-varying disaster risk. The proposed affine approximation performs similarly to global solution methods in many applications; risk pricing is accurate at all investment horizons, thereby capturing the main properties of investors' marginal utility of wealth and measures of welfare costs of fluctuations. ${ }^{3}$
\end{abstract}

Keywords: Perturbation methods, Risky steady state, Macroeconomic uncertainty, Intertemporal risk prices, Risk-return tradeoff

JEL classification: C63, G12, E32, E44.

\footnotetext{
${ }^{1}$ Banque de France, pierlauro.lopez@banque-france.fr.

${ }^{2}$ Federal Reserve Board.

3 A flexible computer code is available on the authors' website. This paper was previously circulated under the titles "Exponential-Affine Approximations of Macro-Finance Models" and "Entropy-Based Approximations of Dynamic Equilibrium Models". We would like to thank Jarda Borovicka, Luca Guerrieri, Patrick Kehoe, Michel Juillard, Serguei Maliar, Aytek Malkhozov, and Inna Tsener for very useful comments, as well as seminar participants at the 2016 and 2018 meetings of the EEA/ESEM, the Econometric Society 2016 North American summer meeting, the 2016 meeting of the Society for Computational Economics, the 2017 meetings of the Society for Nonlinear Dynamics and Econometrics, the 2017 CEPR/MMCN conference, the Federal Reserve Board, and the Bank of France for comments and discussions. The views presented here are solely those of the authors and do not necessarily represent those of the Federal Reserve System or the Eurosystem.
}

Working Papers reflect the opinions of the authors and do not necessarily express the views of the Banque de France. This document is available at publications.banque-france.fr/en 


\section{NON-TECHNICAL SUMMARY}

People make decisions under uncertainty and are sensitive to risk. Accordingly, financial prices and the macroeconomy reflect, and move with, the exposure of future cashflows to risky events and the compensation for risk commanded by investors. Examples of the ingredients economists use to capture these facts include risk appetite that changes with the state of the economy, and a varying probability of the realization of some disastrous event such as a financial crisis or war. The success of these ingredients relies on solution methods that can capture correctly their dynamic equilibrium implications.

Numerical methods that reveal the global solution of a model are used increasingly by economists. Nevertheless, these methods are typically computationally intensive-a cost particularly large in estimation-and offer limited analytic insight into the main economic channels that drive the solution. Analytic tractability is key to understand the mechanics of a model. For one thing, it helps identifying the role of different assumptions and parameters in driving particular results. Furthermore, it helps talking about existence, uniqueness, or multiplicity of equilibrium dynamics.

In this context, perturbation methods provide analytic insight into the local structure of a model around a specific point. But for these properties to be meaningful, the expansion point should be a point the model actually fluctuates around. In fact, the conventional expansion point for perturbation methods - the deterministic steady state—and local dynamics around it are often inaccurate approximations of a model's implications when risk matters. Higher-order perturbations do not always help and, in any event, analytic insight is quickly lost as the order of approximation increases.

We propose a simple risk-adjusted linear approximation to solve a large class of dynamic models with time-varying and non-Gaussian risk. Our approach generalizes loglinear-lognormal approximations commonly used in the finance literature, and we show that it coincides with firstorder perturbations around the risky steady state recently developed in the macro literature. Therefore, we provide a formal foundation for approximation methods that remained so far heuristic, and we unify coexisting theories of risk-adjusted linearizations. Two strands of literature that developed independently are actually one and the same.

We make two main contributions. First, we generalize affine approximations. We extend risk adjustments to non-Gaussian distributed shocks using relative entropy-a generalized notion of variation-and the cumulant generating function of shocks. And we discuss the main features of this approximation that determine its accuracy.

Second, we root in formal ground our unified theory of risk-adjusted linearizations based on the implicit function and Taylor theorems. Our approach provides explicit formulas for the approximation coefficients, clarifies when the risky steady state is defined uniquely, and characterizes local existence and uniqueness of the approximate solution. Therefore, we are the first to provide a complete description of first-order perturbations around the risky steady state. 


\section{Equilibrium riskless bond yield and wealth in the Campbell-Cochrane model. Markers denote mean values under the different solution methods: affine approximation, conventional perturbations, and global solution.}
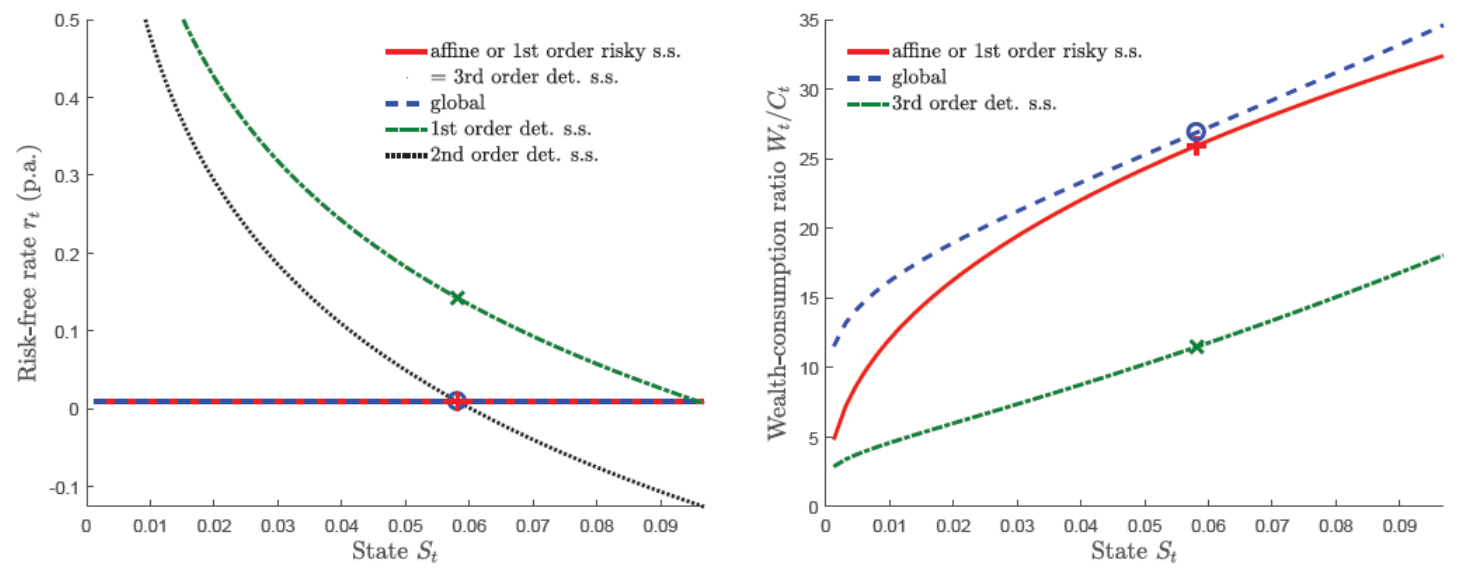

The top figure illustrates the importance of risk corrections in a basic example. With habit formation risk aversion is time-varying, and low-order perturbations around the deterministic steady state are inappropriate. They fail to capture the key role of precautionary savings in providing greater incentives to save in a risk-free security, especially during recessions when people are unwilling to take on more risk and invest in risky projects. Conventional third-order perturbations recover the global structure of the equilibrium risk-free rate but remain inappropriate to characterize equilibrium wealth. In contrast, affine perturbations offer an accurate description of local equilibrium dynamics.

Thus, affine approximations seem to be an appropriate perturbation choice to gain analytic understanding of the macroeconomic forces that drive macroeconomic quantities, asset prices, and welfare costs of fluctuations. To popularize these methods we provide a user-friendly computer code, flexible enough for application to most DSGE models.

\section{Linéarisations ajustées au risque des modèles dynamiques d'équilibre générale}

Nous proposons une simple approximation linéaire ajustée au risque pour résoudre une grande classe de modèles dynamiques à risque non Gaussien et variant dans le temps. Notre approche généralise les approximations affines lognormales couramment utilisées dans la littérature et peut être considérée comme une perturbation de premier ordre autour de l'état stationnaire risqué. Par conséquent, nous unifions les théories existantes. Nous formalisons des méthodes d'approximation qui sont restées jusqu'à présent heuristiques, et nous dérivons des formules explicites pour caractériser ces aproximations ainsi que les conditions de leur existence et unicité. Les approximations affines ne sont pas imbriquées dans les perturbations conventionnelles. Nous appliquons cette technique à des modèles présentant des habitudes à la Campbell-Cochrane, des préférences récursives et des risques de catastrophe. L'approximation affine proposée est comparable aux méthodes de résolution globale par de nombreux aspects.

Mots-clés: Méthodes de perturbation, État stationnaire risqué, Incertitude macroéconomique, Prix du risque intertemporel, arbitrage risque-rendement

Les Documents de travail reflètent les idées personnelles de leurs auteurs et n'expriment pas nécessairement la position de la Banque de France. Ce document est disponible sur publications.banque-france.fr 


\section{Introduction}

Variation in risk sensitivity is a central ingredient in modern dynamic equilibrium models. It is necessary to capture asset pricing facts and to study the effects of macroeconomic uncertainty. Accordingly, in the last twenty years researchers have explored features such as time-varying risk aversion, risk-sensitive preferences, stochastic volatility, variable disaster risk, and portfolio choice under uncertain asset returns. ${ }^{1}$ Models in which risk plays an important role present a challenge for extant solution techniques. Projection methods are accurate but computationally intensive and offer limited analytic insight. Higher-order perturbations around the deterministic steady state have similar disadvantages and can misrepresent the model's implications when non-analytic functions are involved. Finally, the deterministic steady state can be an invalid expansion point. ${ }^{2}$

This paper proposes an affine approximation technique to solve and simulate dynamic stochastic general equilibrium models that includes a risk adjustment in equilibrium prices and quantities. Two strains of literature have dealt separately with this problem. First, the macro-finance literature has used affine risk adjustments based on lognormality at least since Hansen and Singleton (1983), as they facilitate an analytic understanding of the asset pricing implications of the model and the use of fast filtering techniques; yet they remain limited in scope and lack a formal justification based on perturbation theory. ${ }^{3}$ Second, a recent literature has been exploring linear approximations around the risky steady state; yet their relationship with standard risk-adjusted linearizations has not been explored and a characterization of their exact solution, uniqueness, and local stability properties has so far proved elusive. ${ }^{4,5}$

We make two main contributions. First, we generalize affine approximations. We extend risk adjustments to non-Gaussian distributed shocks using relative entropy as the measure of dispersion and the cumulant generating function of shocks, and we ensure consistent risk corrections. ${ }^{6}$

\footnotetext{
${ }^{1}$ For example, among many others, Campbell and Cochrane (1999); Chamberlain and Wilson (2000); Bansal and Yaron (2004); Binsbergen et al. (2012); Gourio (2012); Rudebusch and Swanson (2012); Fernández-Villaverde et al. (2011); Wachter (2013); Fernández-Villaverde et al. (2015b); Lopez et al. (2015); Kehoe et al. (2018). See also Cochrane (2011) and Bloom (2014).

${ }^{2}$ As in portfolio choice problems in small open economies (section 3.3 and Schmitt-Grohé and Uribe, 2003).

${ }^{3}$ Examples of loglinear-lognormal methods applied to asset pricing include Campbell (1993); Jermann (1998); Lettau and Uhlig (2000); Bansal and Yaron (2004); Uhlig (2007); Alvarez et al. (2007); Bekaert et al. (2010); Kaltenbrunner and Lochstoer (2010); Verdelhan (2010); Dew-Becker (2014); Malkhozov (2014); Backus et al. (2015); Chen and Palomino (2018) among many others.

${ }^{4}$ The risky steady state is the point where agents choose to stay while expecting shocks in the future and when ex-post realized shocks are zero (Coeurdacier et al., 2011; Juillard, 2011). In contrast, the deterministic steady state is the point where agents choose to stay while knowing that future realized shocks are zero.

${ }^{5}$ Coeurdacier et al. (2011) and Juillard (2011) are the first to study explicitly perturbations around the risky steady state. Meyer-Gohde (2016) extends the analysis to perturbations around the ergodic mean.

${ }^{6}$ In particular, extant affine approaches (Malkhozov, 2014, is the most recent formalization of the lognormal affine method) accommodate dynamic risk corrections only when the conditional distribution of innovations in state variables is known a priori. In contrast, we accommodate dynamic risk corrections even when risk is endogenous in the sense that the distribution of innovations in state variables is known only after the model is solved. (Examples include production economies with habits and portfolio choice under uncertainty in small open economies.) It follows that our approximation is continuous at parametrizations at which production economies coincide with endowment economies, a natural consistency requirement.
} 

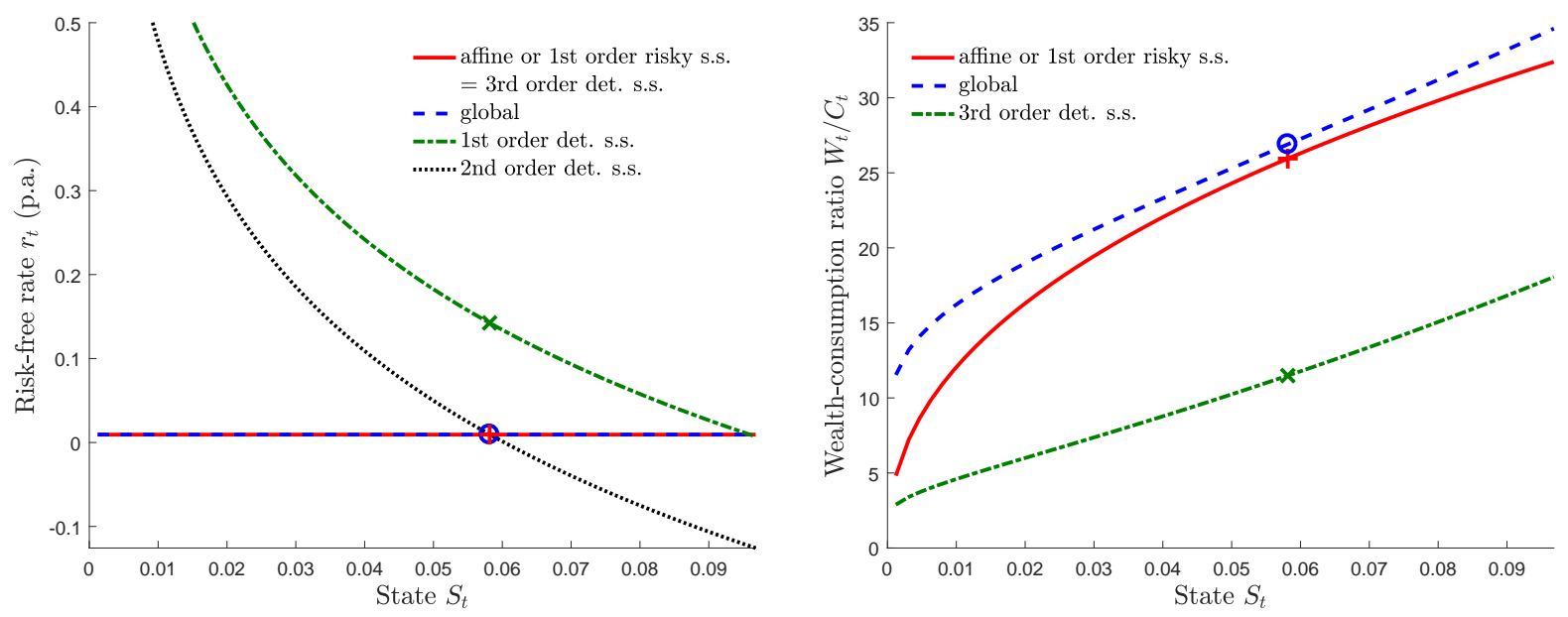

Figure 1: Equilibrium risk-free rate and price-dividend ratio of the consumption portfolio as a function of the state of the economy in Campbell and Cochrane (1999). Markers denote mean values under the different solution methods. Affine approximation, perturbations around the deterministic steady state of up to third order, and global solution.

Second, we provide a unified theory of risk-adjusted linearizations rooted in formal ground based on the implicit function and Taylor theorems. We show that affine approximations coincide with first-order perturbations around the risky steady state, thereby reconciling the two strands of literature. Equally important, our approach provides explicit formulas for the approximation coefficients, clarifies when the risky steady state is defined uniquely, and characterizes local existence and uniqueness of the approximate solution by generalizing Blanchard and Kahn (1980) saddle-path conditions. Therefore, we are the first to provide a complete description of first-order perturbations around the risky steady state.

Figure 1 illustrates the importance of risk corrections in a basic example: the pricing of a risk-free bond and of the wealth-consumption ratio in the Campbell and Cochrane (1999) habit model. (Sections 2 and 3 elaborate this example.) Low-order perturbations around the deterministic steady state are inappropriate. Conventional third-order perturbations recover the global solution for the risk-free rate but are inappropriate to characterize equilibrium wealth. In contrast, affine approximations - or, equivalently, linearizations around the risky steady state - are close, when not identical, to the global solution around the expansion point.

Generically, we show that our approximation is not nested in deterministic steady state perturbations of arbitrary order. In fact, the deterministic steady state may not even be well-defined in examples in which risk-adjusted perturbations are. ${ }^{7}$

Affine approximations are particularly appealing by their ability to provide analytic solutions that facilitate an intuitive understanding of the macroeconomic forces that drive asset prices, investors' marginal utility of wealth, and welfare. We use our risk-sensitive affine approximation to

\footnotetext{
${ }^{7}$ Malkhozov (2014) suggests that affine approximations are second-order perturbations around the deterministic steady state in which dynamic second-order terms are disregarded, yet we show that the connection to perturbations is actually exact.
} 
provide pricing formulas in the affine class for equilibrium term structures — and hence for all related claims, including wealth portfolios and welfare costs of fluctuations (see Alvarez and Jermann, 2004; Lopez, 2014) — as well as for some major diagnostic decompositions of the asset pricing properties of a model - including Hansen and Jagannathan (1991) and Backus, Chernov, and Zin (2014) bounds, Alvarez and Jermann (2005) and Hansen and Scheinkman (2009) decompositions, and Borovicka and Hansen (2014) elasticities.

To test the accuracy of our method in a challenging context we consider general equilibrium models with nonlinear habits and models with recursive utility and a risk of rare disasters. Projection methods are notoriously required to find the global solution under nonlinear habits; rare disasters are main examples of non-Gaussian exogenous shocks.

We first test the performance of our approximation procedure in endowment economies. We consider the habit formation model of Campbell and Cochrane (1999) and the disaster risk model of Wachter (2013). Our approximation is accurate in solving for risk premia and volatilities of equities and bonds at both short and long durations, and produces continuous disaster-risk corrections.

We then turn to a production economy. The real business cycle model of Jermann (1998) with Campbell-Cochrane habits recently explored by Chen (2017) is appropriate for testing the accuracy of our solution in an environment where consumption risk is endogenous, while habits and capital adjustment costs generate volatile stock market-book ratios. In this application the full nonlinear solution is computationally expensive, while our generalized affine approximation yields a fast and tractable solution with good accuracy.

Finally, we provide a user-friendly computer code for application to most DSGE models.

The rest of the paper is structured as follows. Section 2 describes our affine approximation heuristically. Section 3 illustrates it in simple examples. Section 4 presents formal results motivating our method. Section 5 inspects approximate risk pricing formulas. Section 6 applies the method to main examples in the literature and tests its accuracy against competing solutions. Section 7 concludes. Proofs are in the appendix.

\section{Approximation method: Heuristic algorithm}

\subsection{Basic examples}

Suppose we want to price the equilibrium log risk-free rate $(r)$ described by equation:

$$
0=\ln E_{t} e^{m_{t+1}+r_{t}}
$$

for some stochastic discount factor $m$.

\subsubsection{Nonlinear habits}

Consider the stochastic discount factor given by $m_{t+1}=\ln (\beta)-\gamma \Delta c_{t+1}-\gamma \Delta s_{t+1}$, for some parameters $\beta \in(0,1)$ and $\gamma>0$, and by the law of motion of the state vector:

$$
\hat{s}_{t+1}=\phi \hat{s}_{t}+\Lambda\left(\hat{s}_{t}\right) \sigma \varepsilon_{t+1}
$$


for some nonlinear function $\Lambda\left(\hat{s}_{t}\right)$ and random-walk consumption $c_{t+1}=\mu+c_{t}+\sigma \varepsilon_{t+1}$, with $\varepsilon_{t} \sim \operatorname{Niid}(0,1)$. (The familiar reader will recognize the stochastic discount factor in Campbell and Cochrane, 1999.)

The idea of affine approximations is to conjecture an affine solution in the state $r_{t}=r+\psi_{r} \hat{s}_{t}$, and to rewrite the Euler equation as the sum of a certainty equivalent and a variance term by exploiting the normality of shocks:

$$
\begin{aligned}
0=E_{t} m_{t+1}+r_{t}+\frac{1}{2} \operatorname{var}_{t}\left(m_{t+1}\right) & =\ln \left(\beta e^{-\gamma \mu}\right)+\gamma(1-\phi) \hat{s}_{t}+r+\psi_{r} \hat{s}_{t}+\frac{1}{2} \operatorname{var}_{t}\left(-\gamma \Delta c_{t+1}-\gamma \Delta s_{t+1}\right) \\
& =\ln \left(\beta e^{-\gamma \mu}\right)+\gamma(1-\phi) \hat{s}_{t}+r+\psi_{r} \hat{s}_{t}+\frac{\gamma^{2}\left[1+\Lambda\left(\hat{s}_{t}\right)\right]^{2} \sigma^{2}}{2}
\end{aligned}
$$

Linearizing any remaining nonlinearities and matching coefficients identifies the solution as:

$$
r=-\ln \left(\beta e^{-\gamma \mu}\right)-\frac{\gamma^{2}[1+\Lambda(0)]^{2} \sigma^{2}}{2}, \quad \psi_{r}=-\gamma(1-\phi)-\gamma^{2}[1+\Lambda(0)] \Lambda_{1}(0) \sigma^{2}
$$

where the last term in both coefficients is a risk correction that reflects precautionary saving.

\subsubsection{Long-run risk}

Consider the Epstein-Zin discount factor given by $m_{t+1}=\ln (\beta)-\rho \Delta c_{t+1}-(\gamma-\rho)\left(v_{t+1}-x_{t}\right)$, with rate of time preference $\beta$, elasticity of intertemporal substitution $1 / \rho$, and risk aversion coefficient $\gamma$, for preferences $(v)$ and certainty equivalent $(x)$ :

$$
v_{t}=c_{t}+\frac{1}{1-\rho} \ln \left(1-\beta+\beta e^{(1-\rho)\left(x_{t}-c_{t}\right)}\right), \quad x_{t}=\frac{1}{1-\gamma} \ln E_{t} e^{(1-\gamma) v_{t+1}}
$$

The consumption process $c_{t+1}=\mu+c_{t}+u_{t}+\sigma \varepsilon_{t+1}^{c}, u_{t+1}=\rho_{u} u_{t}+\phi \sigma \varepsilon_{t+1}^{u}$, where $\varepsilon_{t}^{c}, \varepsilon_{t}^{u} \sim \operatorname{Niid}\left(0, I_{2}\right)$, displays long-run risk as in Bansal and Yaron (2004).

In this example an affine conjecture $r_{t}=r+\psi_{r} u_{t}, v_{t}-c_{t}=v c+\psi_{v} u_{t}$, and $x_{t}-c_{t}=x c+\psi_{x} u_{t}$ implies the conditional normality of preferences, hence the certainty equivalent:

$$
\begin{aligned}
x_{t}-c_{t} & =E_{t}\left(v_{t+1}-c_{t+1}\right)+E_{t} \Delta c_{t+1}+\frac{(1-\gamma)}{2} \operatorname{var}_{t}\left(v_{t+1}\right) \\
& =v c+\psi_{v} \rho_{u} u_{t}+\mu+u_{t}+\frac{(1-\gamma)}{2}\left(1+\psi_{v} \phi\right)^{2} \sigma^{2}=x c+\psi_{x} u_{t}
\end{aligned}
$$

and the equilibrium risk-free rate:

$$
\begin{aligned}
r_{t} & =-\ln (\beta)+\rho E_{t} \Delta c_{t+1}+(\gamma-\rho) E_{t}\left(v_{t+1}-x_{t}\right)-\frac{1}{2} \operatorname{var}_{t}\left[\rho c_{t+1}+(\gamma-\rho) v_{t+1}\right] \\
& =-\ln (\beta)+\rho\left(\mu+u_{t}\right)+\frac{(\gamma-\rho)(\gamma-1)\left(1+\psi_{\nu} \phi\right)^{2} \sigma^{2}}{2}-\frac{\left[\gamma+(\gamma-\rho) \psi_{\nu} \phi\right]^{2} \sigma^{2}}{2}=r+\psi_{r} u_{t}
\end{aligned}
$$

The quadratic terms in the expression related to conditional variances represent risk corrections that reflect precautionary saving as well as a preference for timing in the resolution of uncertainty. 
To identify the affine solution it is sufficient to linearize the remaining nonlinearities in

$$
v_{t}-c_{t} \approx \frac{1}{1-\rho} \ln \left(1-\beta+\beta e^{(1-\rho) x c}\right)+\beta e^{(1-\rho) x c} \psi_{x} u_{t}=v c+\psi_{v} u_{t}
$$

around the conjectured mean values, and match coefficients to identify the vector $\left[r, \psi_{r}, v c, \psi_{v}, x c, \psi_{x}\right]$.

We can generalize this intuitive line of reasoning.

\subsection{General framework}

We aim at characterizing the solution for jump variables $y_{t} \in \mathbb{R}^{n_{y}}$ and states $z_{t} \in \mathbb{R}^{n_{z}}$ of the dynamic system of equilibrium conditions with generic form:

$$
\begin{aligned}
0 & =\ln E_{t} e^{f\left(y_{t}, z_{t}, y_{t+1}, z_{t+1}\right)}, \quad f\left(y_{t}, z_{t}, y_{t+1}, z_{t+1}\right) \doteq h\left(y_{t}, z_{t}\right)+f_{3} y_{t+1}+f_{4} z_{t+1} \\
z_{t+1} & =g\left(y_{t}, z_{t}\right)+\lambda\left(z_{t}\right)\left(y_{t+1}-E_{t} y_{t+1}\right)+\sigma\left(z_{t}\right) \varepsilon_{t+1}
\end{aligned}
$$

where $\lambda\left(z_{t}\right)\left(y_{t+1}-E_{t} y_{t+1}\right)$ describes heteroskedastic endogenous risk that depends on innovations in jump variables and $\sigma\left(z_{t}\right) \varepsilon_{t+1}$ is exogenous risk. Operator $\ln E_{t} e^{[\cdot]}$ is applied elementwise to a vector-valued map, with $E_{t}$ the expectations operator conditioned on the history up to time- $t$ of state variables. Functions $f: \mathbb{R}^{2 n_{y}+2 n_{z}} \rightarrow \mathbb{R}^{n_{y}}, h: \mathbb{R}^{n_{y}+n_{z}} \rightarrow \mathbb{R}^{n_{y}}, g: \mathbb{R}^{n_{y}+n_{z}} \rightarrow \mathbb{R}^{n_{z}}, \lambda: \mathbb{R}^{n_{z}} \rightarrow \mathbb{R}^{n_{z} \times n_{y}}$ and $\sigma: \mathbb{R}^{n_{z}} \rightarrow \mathbb{R}^{n_{z} \times n_{\varepsilon}}$ are differentiable. We denote by $f_{i}, g_{i}, \ldots$ the derivatives of $f, g, \ldots$ with respect to the $i$ th argument; function $f$ is linear in $y_{t+1}$ and $z_{t+1}$. The equilibrium conditions of most DSGE models can be cast into this framework after suitable redefinition of variables.

Exogenous shocks $\varepsilon_{t} \in \mathbb{R}^{n_{\varepsilon}}$ form a martingale difference sequence with distribution described by the differentiable, conditional cumulant generating function (ccgf) $:^{8}$

$$
\kappa\left[\alpha\left(z_{t}\right) ; z_{t}\right] \doteq \ln E_{t} e^{\alpha\left(z_{t}\right)^{\prime} \varepsilon_{t+1}}, \quad \text { for any differentiable map } \alpha: \mathbb{R}^{n_{z}} \rightarrow \mathbb{R}^{n_{\varepsilon}}
$$

\subsection{Affine approximation}

We are interested in approximating with an affine map the solution of system (1) for jump and state variables. Without loss of generality, we rewrite forward-looking equations as:

$$
0=h\left(y_{t}, z_{t}\right)+f_{3} E_{t} y_{t+1}+f_{4} E_{t} z_{t+1}+\mathcal{V}_{t}\left(e^{f_{3} y_{t+1}+f_{4} z_{t+1}}\right)
$$

where $\mathcal{V}_{t}\left(e^{x_{t+1}}\right) \doteq \ln E_{t} e^{x_{t+1}}-E_{t} x_{t+1}$ is a relative entropy measure-a nonnegative measure of dispersion that generalizes variance. The presence of entropy breaks certainty equivalence.

We are looking for an affine solution $y_{t}=y+\Psi\left(z_{t}-z\right)$ with unknown coefficients $y, \Psi$, and $z$. If $I_{n_{z}}-\lambda\left(z_{t}\right) \Psi$ is invertible, then innovations to the state vector have the approximate form:

$$
z_{t+1}-E_{t} z_{t+1}=\lambda\left(z_{t}\right) \Psi\left(z_{t+1}-E_{t} z_{t+1}\right)+\sigma\left(z_{t}\right) \varepsilon_{t+1}=\left(I_{n_{z}}-\lambda\left(z_{t}\right) \Psi\right)^{-1} \sigma\left(z_{t}\right) \varepsilon_{t+1}
$$

\footnotetext{
${ }^{8}$ For example, if $\varepsilon_{t} \sim \operatorname{Niid}(0, I)$, one has $\kappa\left[\alpha\left(z_{t}\right) ; z_{t}\right]=.5 \operatorname{diag}\left[\alpha\left(z_{t}\right) \alpha\left(z_{t}\right)^{\prime}\right]$. In the special case of Gaussian shocks, a constant function $\lambda$ and linear functions $h$ and $g$, our affine approximation reduces to the one in Malkhozov (2014).
} 
In this context, rational expectations consistent with the affine solution imply the existence of a nonnegative function $\widetilde{\mathcal{V}}: \mathbb{R}^{n_{z}} \rightarrow \mathbb{R}_{+}^{n_{y}}$ of the state vector:

$$
\widetilde{\mathcal{V}}\left(z_{t}\right) \doteq \mathcal{V}_{t}\left(e^{\left(f_{3} \Psi+f_{4}\right) z_{t+1}}\right)=\kappa\left[\left(f_{3} \Psi+f_{4}\right)\left(I_{n_{z}}-\lambda\left(z_{t}\right) \Psi\right)^{-1} \sigma\left(z_{t}\right) ; z_{t}\right]
$$

where the connection with the ccgf follows from the properties of entropy.

Finally, we linearize equations (2) and (3) around the point $\left[y_{t} ; z_{t}\right]=[y ; z]$ as:

$$
\begin{aligned}
0 & =h(y, z)+f_{1}\left(y_{t}-y\right)+f_{2}\left(z_{t}-z\right)+f_{3} E_{t} y_{t+1}+f_{4} E_{t} z_{t+1}+\widetilde{\mathcal{V}}(z)+\widetilde{\mathcal{V}}_{1}(z)\left(z_{t}-z\right) \\
E_{t} z_{t+1} & =g(y, z)+g_{1}\left(y_{t}-y\right)+g_{2}\left(z_{t}-z\right)
\end{aligned}
$$

with the notation $f_{i} \doteq f_{i}(y, z, y, z)$ and $g_{i} \doteq g_{i}(y, z)$.

We can now match coefficients to identify the conjectured affine solution:

$$
\begin{aligned}
y_{t} & =y+\Psi\left(z_{t}-z\right) \\
z_{t+1} & =z+g_{1}\left(y_{t}-y\right)+g_{2}\left(z_{t}-z\right)+\left(I_{n_{z}}-\lambda\left(z_{t}\right) \Psi\right)^{-1} \sigma\left(z_{t}\right) \varepsilon_{t+1}
\end{aligned}
$$

where the unknowns $[y, z, \Psi]$ solve the system of equations:

$$
\begin{aligned}
& 0=g(y, z)-z \\
& 0=h(y, z)+f_{3} y+f_{4} z+\widetilde{\mathcal{V}}(z) \\
& 0=f_{1} \Psi+f_{2}+\left(f_{3} \Psi+f_{4}\right)\left(g_{1} \Psi+g_{2}\right)+\widetilde{\mathcal{V}}_{1}(z)
\end{aligned}
$$

Crucially, the entropy terms represent risk corrections to an otherwise standard linearization.

Heuristically, general affine approximations can be summarized in the following algorithm:

Algorithm. With system (1) as a starting point, proceed stepwise:

Step 1. Write expectations as the sum of a certainty-equivalent and an entropy terms [(2)].

Step 2. Conjecture a solution affine in the states and use it to characterize entropy [(3)].

Step 3. Identify the affine solution (4) by solving matrix equation (5).

\subsection{Discussion}

The solution of matrix equation (5) deserves some comment. Constant terms $[y, z]$ and dynamic coefficient $\Psi$ are identified jointly at the end of the algorithm. Expression (5) includes nonlinear matrix equations in the unknown coefficients that are amenable to straightforward Newton-type numerical solution methods. ${ }^{9}$ However, these matrix equations are sufficiently nonlinear to allow for multiple solutions and to complicate the characterization of the local uniqueness of the constant terms and of the determinacy of the affine solution's dynamics. Section 4 provides this characterization by drawing a link between affine approximations and perturbations around the risky steady state. In particular, constant terms $[y, z]$ acquire an appealing economic interpretation as a risky steady state.

\footnotetext{
${ }^{9}$ The online appendix discusses simple numerical algorithms to solve matrix equation (5).
} 
There are two key implicit assumptions in representation (1). First, forward-looking arguments of the expectations operator must be strictly positive - a necessary property for a connection with entropy. While this assumption is not without loss of generality, most problems can be rewritten appropriately by splitting the argument into strictly positive components.

Second, function $f$ must be linear in $y_{t+1}$ and $z_{t+1}$. This assumption is not restrictive-one can always define new variables to impose the linear structure. In fact, there are infinite ways to represent a model in this form whenever a forward-looking difference equation is present. Although the way we choose has no consequences for conventional riskless perturbations, the choice matters for affine approximations because it determines which variables are approximated as affine in the states, and that in turn affects entropy calculations - a point so far unrecognized by the literature. How should we select the best specification? Intuitively, you need to pick the vector $f_{3} y_{t}+f_{4} z_{t}$ whose exact solution is closest to affine. In practice, you should select the one whose associated affine approximation minimizes the difference equation's Euler equation error. (The example in section 3.1 illustrates these points.)

There are also two minor assumptions in the representation of innovations that can be relaxed easily. First, we can generalize representation (2) to handle a dependence also on jump variables $y_{t}$ of functions $\lambda$ and $\sigma$-that would be replaced by functions $\widetilde{\lambda}\left(y_{t}, z_{t}\right)$ and $\widetilde{\sigma}\left(y_{t}, z_{t}\right)$. In that case define $\lambda\left(z_{t}\right)=\widetilde{\lambda}\left(y+\Psi\left(z_{t}-z\right), z_{t}\right)$ and $\sigma\left(z_{t}\right)=\widetilde{\sigma}\left(y+\Psi\left(z_{t}-z\right), z_{t}\right)$ and proceed as before. Second, when describing the dynamics of the state vector in (4), one may also choose to approximate the volatility of innovations $\left(I_{n_{z}}-\lambda\left(z_{t}\right) \Psi\right)^{-1} \sigma\left(z_{t}\right)$ around $z_{t}=z$. This approximation would not affect the affine coefficients - it would only affect simulations from the model — but it has no practical advantage because the underlying maps $\lambda$ and $\sigma$ in leading examples have Taylor series with a small radius of convergence. Approximations of those maps can result in spurious dynamics, with an inaccurate representation of tail regions of the state space that matter most for pricing.

\section{Three didactic examples}

We illustrate our affine approximation in the context of three simple models, and compare it with conventional perturbations. We start by pricing a risk-free bond and wealth in the Gaussian endowment economy of Campbell and Cochrane (1999). (This example extends 2.1.1.) We then show how to handle a non-Gaussian disaster component by pricing a risk-free bond and wealth in the model of Wachter (2013). (This example extends 2.1.2.) Finally, we show how a savings problem in a small open economy with an exogenous risk-free bond associates with invalid deterministic steady state perturbations and yet a valid affine approximation.

(In what follows lower-case letters and hat variables will denote respectively logarithms and log deviations from the expansion point.)

\subsection{Habit formation}

A representative consumer with Campbell and Cochrane (1999) preferences:

$$
E_{0} \sum_{t=0}^{\infty} \beta^{t} \frac{\left(C_{t}-X_{t}\right)^{1-\gamma}-1}{1-\gamma}
$$


lives in an endowment economy that describes the equilibrium risk-free rate $(r)$ and the log wealthconsumption ratio $(w c)$ as a function of two state variables - the consumption process $(C)$ and a process for surplus consumption $(S \doteq 1-X / C)$ relative to an external habit level $(X)$. Parameter $\beta$ is the rate of time preference and $1 / \gamma$ is the elasticity of intertemporal substitution.

This endowment economy is described by the pricing equation for the risk-free rate (priced in section 2.1.1) and by the pricing equation for the wealth portfolio $W_{t}=C_{t}+E_{t} M_{t+1} W_{t+1}$ :

$$
\begin{aligned}
e^{w c_{t}} & =1+E_{t} e^{m_{t+1}+\Delta c_{t+1}+w c_{t+1}} \\
& =E_{t} e^{m_{t, t+N}+c_{t+N}-c_{t}+w c_{t+N}}+\sum_{n=0}^{N-1} E_{t} e^{m_{t, t+n}+c_{t+n}-c_{t}}
\end{aligned}
$$

where $w c_{t} \doteq w_{t}-c_{t}$ is the log wealth-consumption ratio and $m_{t, t+n}=\sum_{j=1}^{n} m_{t+j}$ is the $n$-period stochastic discount factor. Because it is a forward-looking difference equation, equation (6) can be written as (7), so there are infinite ways to cast it into form (2). Namely, for a given $N>0$, we must solve the system of $2 N$ equations:

$$
w c_{t}=\ln \left(e^{r c_{t}^{(N)}}+\sum_{n=0}^{N-1} e^{p c_{t}^{(n)}}\right), \quad p c_{t}^{(n)}=\ln E_{t} e^{m_{t+1}+\Delta c_{t+1}+p c_{t+1}^{(n-1)}}, \quad r c_{t}^{(n)}=\ln E_{t} e^{m_{t+1}+\Delta c_{t+1}+r c_{t+1}^{(n-1)}}
$$

with boundary conditions $p c_{t}^{(0)}=0$ and $r c_{t}^{(0)}=w c_{t}$, where $p c_{t}^{(n)}$ describes the log price-consumption ratio of the $n$th consumption strip, i.e., a claim to $n$-period ahead consumption, and $r c_{t}^{(n)}$ is the $\log$ value of a claim to $n$-period ahead wealth as a fraction of consumption.

Including difference specification (6) —or (7) with $N=1$ - among the equilibrium conditions implies approximating the price of the sum of strips as conditionally lognormal. Including specification (7) for $N \rightarrow \infty$ implies approximating each strip price as conditionally lognormal. Since the sum of lognormals is generically not a lognormal, and in contrast with conventional riskless perturbations, it follows that it matters which specification we choose to approximate.

\subsubsection{Affine approximation}

We use the algorithm in section 2 to obtain an affine approximation of equation (7).

Step 1. Write expectational equations in terms of a certainty-equivalent and entropy:

$$
0=\ln (\beta)+(1-\gamma) E_{t} \Delta c_{t+1}-\gamma E_{t} \Delta s_{t+1}+E_{t} p c_{t+1}^{(n-1)}-p c_{t}^{(n)}+\mathcal{V}_{t}\left(e^{(1-\gamma) \Delta c_{t+1}-\gamma \Delta s_{t+1}+p c_{t+1}^{(n-1)}}\right)
$$

Step 2. Conjecture affine solutions $p c_{t}^{(n)}=p c^{(n)}+\psi^{(n)} \hat{s}_{t}$ and use the Gaussian ccgf to characterize the entropy terms as:

$$
\mathcal{V}_{t}\left(e^{(1-\gamma) \Delta c_{t+1}-\gamma \Delta s_{t+1}+p c_{t+1}^{(n-1)}}\right)=\left(1-\gamma\left[1+\Lambda\left(\hat{s}_{t}\right)\right]+\psi^{(n-1)} \Lambda\left(\hat{s}_{t}\right)\right)^{2} \frac{\sigma^{2}}{2}
$$


Step 3.i. Linearize:

$$
\begin{aligned}
0= & \ln (\beta)+(1-\gamma) E_{t} \Delta c_{t+1}-\gamma E_{t} \Delta s_{t+1}+E_{t} p c_{t+1}^{(n-1)}-p c_{t}^{(n)}+\left(1-\gamma\left[1+\Lambda\left(\hat{s}_{t}\right)\right]+\psi^{(n)} \Lambda\left(\hat{s}_{t}\right)\right)^{2} \frac{\sigma^{2}}{2} \\
\approx & p c^{(n-1)}-p c^{(n)}+\ln \left(\beta e^{(1-\gamma) \mu}\right)+\left(1-\gamma[1+\Lambda(0)]+\psi^{(n-1)} \Lambda(0)\right)^{2} \frac{\sigma^{2}}{2} \\
& +\left[\psi^{(n-1)} \phi-\psi^{(n)}+\gamma(1-\phi)+\left(1-\gamma[1+\Lambda(0)]+\psi^{(n-1)} \Lambda(0)\right)\left(\psi^{(n-1)}-\gamma\right) \Lambda_{1}(0) \sigma^{2}\right] \hat{s}_{t}
\end{aligned}
$$

Step 3.ii. Match coefficients to identify the unknown vector $\left[r ; \psi_{r} ; p c^{(n)} ; \psi^{(n)}\right]$ as:

$$
\begin{aligned}
p c^{(n)} & =p c^{(n-1)}+\ln \left(\beta e^{(1-\gamma) \mu}\right)+\left(1-\gamma[1+\Lambda(0)]+\psi^{(n-1)} \Lambda(0)\right)^{2} \frac{\sigma^{2}}{2} \\
\psi^{(n)} & =\psi^{(n-1)} \phi+\gamma(1-\phi)+\left(1-\gamma[1+\Lambda(0)]+\psi^{(n-1)} \Lambda(0)\right)\left(\psi^{(n-1)}-\gamma\right) \Lambda_{1}(0) \sigma^{2}
\end{aligned}
$$

with boundary condition $p c^{(0)}=\psi^{(0)}=0$.

Analogously, $r c_{t}^{(n)}=r c^{(n)}+\varphi^{(n)} \hat{s}_{t}$ where

$$
\begin{aligned}
& r c^{(n)}=r c^{(n-1)}+\ln \left(\beta e^{(1-\gamma) \mu}\right)+\left(1-\gamma[1+\Lambda(0)]+\varphi^{(n-1)} \Lambda(0)\right)^{2} \frac{\sigma^{2}}{2} \\
& \varphi^{(n)}=\varphi^{(n-1)} \phi+\gamma(1-\phi)+\left(1-\gamma[1+\Lambda(0)]+\varphi^{(n-1)} \Lambda(0)\right)\left(\varphi^{(n-1)}-\gamma\right) \Lambda_{1}(0) \sigma^{2}
\end{aligned}
$$

with $r c^{(0)}=\ln \left(e^{r c^{(N)}}+\sum_{n=0}^{N-1} e^{p c^{(n)}}\right)$ and $\varphi^{(0)}=\left(e^{r c^{(N)}} \varphi^{(N)}+\sum_{n=0}^{N-1} e^{p c^{(n)}} \psi^{(n)}\right) /\left(e^{r c^{(N)}}+\sum_{n=0}^{N-1} e^{p c^{(n)}}\right)$.

It follows that the approximate solution for the log wealth-consumption ratio is:

$$
\begin{aligned}
\hat{s}_{t+1} & =\phi \hat{s}_{t}+\Lambda\left(\hat{s}_{t}\right) \sigma \varepsilon_{t+1} \\
w c_{t} & =\ln \left(e^{r c^{(N)}+\varphi^{(N)} \hat{s}_{t}}+\sum_{n=0}^{N-1} e^{p c^{(n)}+\psi^{(n)} \hat{s}_{t}}\right) \\
& \approx \ln \left(e^{r c^{(N)}}+\sum_{n=0}^{N-1} e^{p c^{(n)}}\right)+\frac{e^{r c^{(N)}} \varphi^{(N)}+\sum_{n=0}^{N-1} e^{p c^{(n)}} \psi^{(n)}}{e^{r c^{(N)}}+\sum_{n=0}^{N-1} e^{p c^{(n)}}} \hat{s}_{t}
\end{aligned}
$$

\subsubsection{Perturbations around the deterministic steady state}

It is instructive to compare our approximation with a conventional third-order approximation, for example as $N \rightarrow \infty$ :

$$
\begin{aligned}
\hat{s}_{t+1} & =\phi \hat{s}_{t}+\Lambda(0) \sigma \varepsilon_{t+1}+\underbrace{\Lambda_{1}(0) \hat{s}_{t} \sigma \varepsilon_{t+1}}_{\text {2nd order term }}+\underbrace{.5 \Lambda_{11}(0) \hat{s}_{t}^{2} \sigma \varepsilon_{t+1}}_{3 \text { rd order term }} \\
r_{t} & =-\ln \left(\beta e^{-\gamma \mu}\right)-\gamma(1-\phi) \hat{s}_{t}-\underbrace{.5 \gamma^{2}[1+\Lambda(0)]^{2} \sigma^{2}}_{\text {2nd order term }}-\underbrace{\gamma^{2}[1+\Lambda(0)] \Lambda_{1}(0) \sigma^{2} \hat{s}_{t}}_{\text {3rd order term }} \\
w c_{t} & =\ln \left(\sum_{n=0}^{\infty} e^{p c_{t}^{(n)}}\right), \quad p c_{t}^{(n)}=n \ln \left(\beta e^{(1-\gamma) \mu}\right)+\bar{\psi}_{1}^{(n)} \hat{s}_{t}+\underbrace{\bar{\psi}_{2}^{(n)}}_{\text {2nd order term }}+\underbrace{\bar{\psi}_{3}^{(n)} \hat{s}_{t}}_{\text {3rd order term }}
\end{aligned}
$$


where $\bar{\psi}_{1}^{(n)}=\gamma\left(1-\phi^{n}\right)$ and:

$$
\begin{array}{ll}
\bar{\psi}_{2}^{(n)}=\bar{\psi}_{2}^{(n-1)}+\left(1-\gamma[1+\Lambda(0)]+\bar{\psi}_{1}^{(n-1)} \Lambda(0)\right)^{2} \frac{\sigma^{2}}{2} & \bar{\psi}_{2}^{(0)}=0 \\
\bar{\psi}_{3}^{(n)}=\bar{\psi}_{3}^{(n-1)} \phi+\left(1-\gamma[1+\Lambda(0)]+\bar{\psi}_{1}^{(n-1)} \Lambda(0)\right)\left(\bar{\psi}_{1}^{(n-1)}-\gamma\right) \Lambda_{1}(0) \sigma^{2}, & \bar{\psi}_{3}^{(0)}=0
\end{array}
$$

A second-order perturbation captures a constant precautionary savings motive. However, it misses the dynamics of the component, which show up only to third order. In this simple example, the equilibrium dependence of decision variables on states under a conventional thirdorder perturbation and under an affine approximation coincide for the risk-free rate but they differ in the equilibrium price of consumption strips. (Proposition 2 formalizes the extent of this distinction between affine approximations and conventional perturbations.) Figure 1 illustrates these properties.

Finally, note that the representation of innovations in state variables is also different by comparing (8) and (11). In fact, the small radius of convergence of function $\Lambda\left(s_{t}\right) \propto \sqrt{1-2 \hat{s}_{t}}$ when approximated around $\hat{s}_{t}=0$ implies that higher-order approximations are especially inaccurate when simulating tail regions of the state space associated with values $\hat{s}_{t}<.5$ that matter most for pricing.

\subsubsection{Numerical example}

We specify sensitivity function $\Lambda\left(\hat{s}_{t}\right)=S^{-1} \sqrt{1-2 \hat{s}_{t}}-1$ and calibrate the model using the values in Campbell and Cochrane (1999) reported in table 1. Figure 2 compares the exact solution to our affine approximation by plotting the map from the value of the state variable (surplus consumption) into the price-dividend ratio of the consumption portfolio.

As discussed in section 2.4, $N$ should be picked to minimize Euler equations errors of the original forward-looking difference equation (6). In this example, affine methods with $N \rightarrow \infty$ offer an accurate approximation around the expansion point, as the log price-consumption ratios of consumption strips are nearly affine in the state vector. To visualize this property, figure 2 plots a solution that adds the approximate prices of strips as in expression (9)—labeled as quasi-affine — and shows that the solution is close to the exact structure globally. ${ }^{10}$

In contrast, conventional third-order perturbations as well as an affine approximation with low $N$ deliver a much less accurate approximation as they capture to a lower extent the nonlinear effect of risk on prices. Overall, perhaps the most remarkable feature of figure 2 is that affine approximations offer an accurate solution; Campbell-Cochrane habits offer a less nonlinear problem than we thought.

\footnotetext{
${ }^{10}$ Moreover, Wachter (2006) warned us of a difference in level apparent when using an insufficiently coarse grid; we see the same effect in figure 2. In this sense, our affine method is similar to a global solution with a relatively small number of grid points, and it can even outperform a global solution with a limited number of grid points.
} 


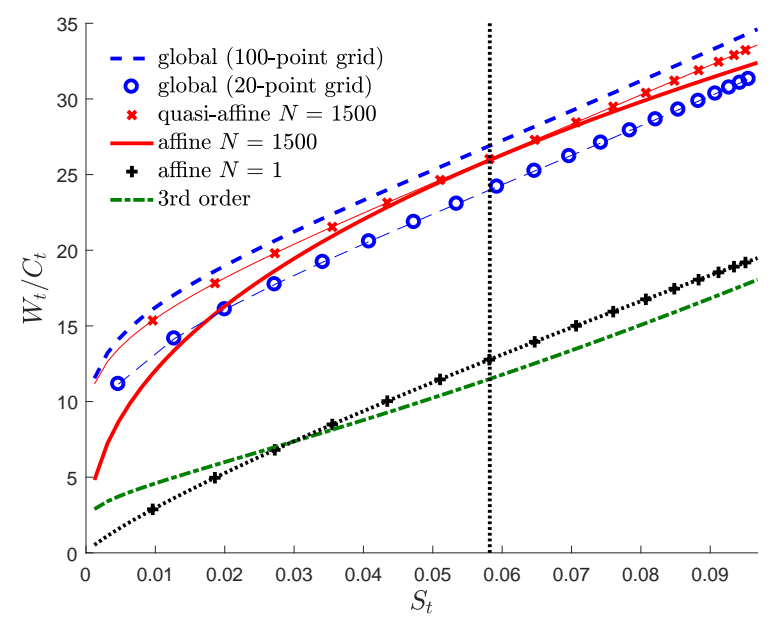

(a) Campbell and Cochrane (1999).

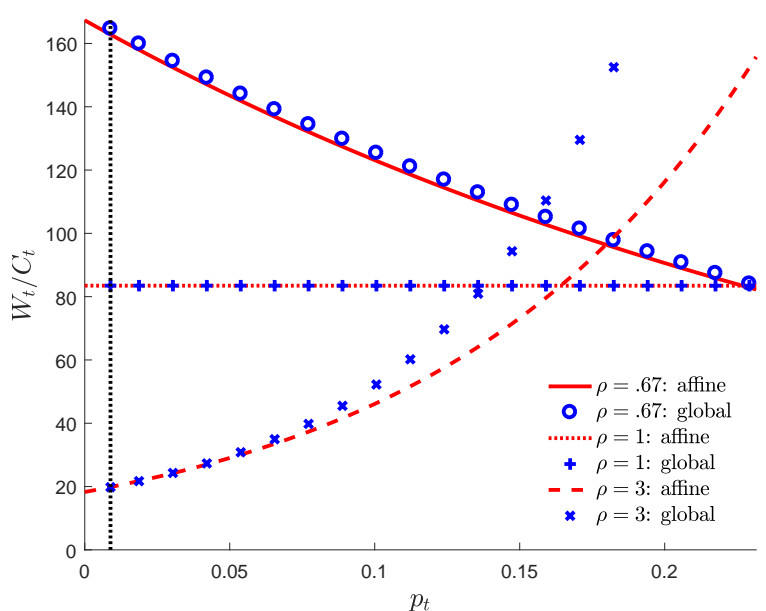

(b) Wachter (2013).

Figure 2: Comparison of solutions for the map of surplus consumption into the wealth-consumption ratio in the models of Campbell and Cochrane (1999) and Wachter (2013). Habit model: Projected solution, affine approximation, and third-order perturbation around the deterministic steady state. 'Affine', 'quasi-affine' and '3rd order' work with expressions (10), (9), and (12). Global solutions use cubic splines collocated over 100 and 20 Chebyshev nodes and 20- and 5-point Gauss-Hermite quadrature, respectively. Rare disaster model: Projected solution and affine approximation for different values of the elasticity of intertemporal substitution. Global solutions use Smolyak collocation of Chebyshev polynomials of up to degree 8 and 10-point Gauss-Hermite quadrature. The vertical, black, dotted line indicates the risky steady-state value of the state.

\begin{tabular}{lcc}
\hline Parameter & Habit formation & Disaster risk \\
\hline Frequency & monthly & quarterly \\
Subjective discount factor, $\beta$ & $.89^{1 / 12}$ & $\exp (-.012 / 4)$ \\
Utility curvature parameter $\gamma$ & 2 & 3 \\
Utility curvature parameter $\rho$ & & $\{1 / 1.5,1,3\}$ \\
Habit persistence, $\phi$ & $.87^{1 / 12}$ & \\
Steady-state surplus consumption ratio, $S$ & .057 & \\
Mean growth rate (in $\%$ ), $\mu$ & $1.89 / 12$ & $2.52 / 4$ \\
Standard deviation of consumption innovations (in $\%), \sigma$ & $1.50 / \sqrt{12}$ & $2.00 / \sqrt{4}$ \\
Average number of disasters per period (in $\%), p$ & & $3.55 / 4$ \\
Mean reversion, $\rho_{p}$ & & $.92^{1 / 4}$ \\
Volatility parameter, $\phi_{p} \sigma$ & & $.0114 / \sqrt{p} / 4$ \\
Impact of disaster, $\theta$ & & -.10 \\
Volatility of disaster impact, $\delta$ & & .09 \\
\hline
\end{tabular}

Table 1: Deep parameters and their calibration. 


\subsection{Disaster risk}

Consider a discrete-time version of the endowment economy in Wachter (2013). Investors have Epstein-Zin recursive preferences described by:

$$
\begin{aligned}
v_{t}-c_{t} & =\frac{\ln (1-\beta)}{1-\rho}+\frac{1}{1-\rho}\left(w_{t}-c_{t}\right) \\
x_{t}-c_{t} & =\frac{1}{1-\rho} \ln \left(e^{w_{t}-c_{t}}-1\right)+\frac{\ln (1-\beta)-\ln (\beta)}{1-\rho} \\
w_{t}-c_{t} & =\ln \left(e^{r c_{t}^{(N)}}+\sum_{n=0}^{N-1} e^{p c_{t}^{(n)}}\right), \quad e^{r c_{t}^{(n)}}=E_{t} e^{m_{t+1}+\Delta c_{t+1}+r c_{t+1}^{(n-1)}}, \quad e^{p c_{t}^{(n)}}=E_{t} e^{m_{t+1}+\Delta c_{t+1}+p c_{t+1}^{(n-1)}}
\end{aligned}
$$

with boundary conditions $p c_{t}^{(0)}=0$ and $r c_{t}^{(0)}=w_{t}-c_{t}$, and $\log$ stochastic discount factor $m_{t+1}=$ $\ln (\beta)-\rho \Delta c_{t+1}+(\rho-\gamma)\left(v_{t+1}-x_{t}\right)$. (Appendix A derives these expressions.)

Investors live in an endowment economy in which log consumption growth has a normal component $\varepsilon^{c}$ as well as a disaster component $\xi$ modeled as a Poisson mixture of normals:

$$
c_{t+1}=\mu+c_{t}+\sigma \varepsilon_{t+1}^{c}-\theta \xi_{t+1}
$$

where $\varepsilon_{t}^{c} \sim \operatorname{Niid}(0,1)$ and $\xi_{t} \mid j_{t} \sim N\left(j_{t}, j_{t} \delta^{2}\right)$, with the number of jumps $j_{t+1} \sim \operatorname{Poisson}\left(p_{t}\right)$, hence $E_{t} \xi_{t+1}=E_{t} j_{t+1}=p_{t}$. We assume that $\varepsilon_{t}^{c}$ and $\varepsilon_{t}^{\xi} \doteq \xi_{t}-E_{t-1} \xi_{t}$ are independent. Jump intensity $p_{t}$ evolves according to the recursive law of motion:

$$
p_{t+1}=\left(1-\rho_{p}\right) p+\rho_{p} p_{t}+\sqrt{p_{t}} \phi_{p} \sigma \varepsilon_{t+1}^{p}
$$

with $\varepsilon_{t}^{p} \sim \operatorname{Niid}(0,1)$ and independent of $\varepsilon_{t}^{c}$ and $\varepsilon_{t}^{\xi}$. It follows that shock $\varepsilon_{t}=\left[\varepsilon_{t}^{c} ; \sqrt{p_{t-1}} \varepsilon_{t}^{p} ; \varepsilon_{t}^{\xi}\right]$ has ccgf: $:^{11}$

$$
\kappa\left(\left[\alpha_{c} ; \alpha_{p} ; \alpha_{\xi}\right] ; p_{t}\right)=\frac{1}{2} \alpha_{c}^{2}+\left[\frac{1}{2} \alpha_{p}^{2}+\left(e^{\alpha_{\xi}+\frac{1}{2} \alpha_{\xi}^{2} \delta^{2}}-1\right)-\alpha_{\xi}\right] p_{t}
$$

As in the previous example, for each $N>0$ there is a way to write model (13) in form (1). We consider $N \rightarrow \infty$, as that value turns out to associate with the most accurate approximation.

\subsubsection{Affine approximation}

Step 1. Write expectational equations in terms of a certainty-equivalent and an entropy terms:

$$
0=\ln (\beta)+(1-\gamma)\left(\mu+\theta p_{t}\right)+(\rho-\gamma)\left(E_{t} v c_{t+1}-x c_{t}\right)+E_{t} p c_{t+1}^{(n-1)}-p c_{t}^{(n)}+\mathcal{V}_{t}\left(e^{(1-\gamma) \Delta c_{t+1}+(\rho-\gamma) v c_{t+1}+p c_{t+1}^{(n-1)}}\right)
$$

\footnotetext{
${ }^{11}$ Entropy is affine in this example. An alternative distribution, $j_{t+1} \sim \operatorname{Bernoulli}\left(p_{t}\right)$, and hence a non-affine ccgf

$$
\kappa\left(\left[\alpha_{c} ; \alpha_{p} ; \alpha_{\xi}\right] ; p_{t}\right)=\frac{1}{2} \alpha_{c}^{2}+\frac{1}{2} \alpha_{p}^{2} p_{t}+\ln \left[1+\left(e^{\alpha_{\xi}+\frac{1}{2} \alpha_{\xi}^{2} \delta^{2}}-1\right) p_{t}\right]-\alpha_{\xi} p_{t},
$$
}

results in a very similar approximation quality. 
Step 2. Conjecture an affine solution $p c_{t}^{(n)}=\alpha_{0}^{(n)}+\alpha_{1}^{(n)} \hat{p}_{t}, w c_{t}=w c+\psi_{w} \hat{p}_{t}, v c_{t}=v c+\psi_{v} \hat{p}_{t}$, and $x c_{t}=x c+\psi_{x} \hat{p}_{t}$ and combine it with the ccgf to characterize the entropy terms:

$$
\mathcal{V}_{t}\left(e^{(1-\gamma) \Delta c_{t+1}+(\rho-\gamma) v c_{t+1}+p c_{l+1}^{(n-1)}}\right)=\frac{(1-\gamma)^{2} \sigma^{2}}{2}+\left[\frac{\left[(\rho-\gamma) \psi_{v}+\alpha_{1}^{(n-1)}\right]^{2} \phi_{p}^{2} \sigma^{2}}{2}+e^{(\gamma-1) \theta+\frac{(\gamma-1)^{2} \theta^{2} \delta^{2}}{2}}-1+(1-\gamma) \theta\right] p_{t}
$$

Step 3.i. Linearize:

$$
\begin{aligned}
0= & \frac{\ln (1-\beta)}{1-\rho}+\frac{w c}{1-\rho}+\frac{1}{1-\rho} \psi_{w} \hat{p}_{t}-v c-\psi_{v} \hat{p}_{t} \\
0= & \frac{\ln (1-\beta)-\ln (\beta)}{1-\rho}+\frac{\ln \left(e^{w c}-1\right)}{1-\rho}+\frac{1}{1-\rho} \frac{e^{w c}}{e^{w c}-1} \psi_{w} \hat{p}_{t}-x c-\psi_{x} \hat{p}_{t} \\
0= & \ln \left(\sum_{n=0}^{\infty} e^{\alpha_{0}^{(n)}}\right)+\frac{\sum_{n=0}^{\infty} e^{\alpha_{0}^{(n)}} \alpha_{1}^{(n)}}{\sum_{n=0}^{\infty} e^{\alpha_{0}^{(n)}}} \hat{p}_{t}-w c-\psi_{w} \hat{p}_{t} \\
0= & \ln \left(\beta e^{(1-\gamma) \mu}\right)+(\rho-\gamma)\left[v c-x c+\left(\psi_{v} \rho_{p}-\psi_{x}\right) \hat{p}_{t}\right]+\alpha_{0}^{(n-1)}-\alpha_{0}^{(n)}+\left(\alpha_{1}^{(n-1)} \rho_{p}-\alpha_{1}^{(n)}\right) \hat{p}_{t} \\
& +\frac{(1-\gamma)^{2} \sigma^{2}}{2}+\left[\frac{\left[(\rho-\gamma) \psi_{v}+\alpha_{1}^{(n-1)}\right]^{2} \phi_{p}^{2} \sigma^{2}}{2}+e^{(\gamma-1) \theta+\frac{(\gamma-1)^{2} \theta^{2} \delta^{2}}{2}}-1\right]\left(p+\hat{p}_{t}\right)
\end{aligned}
$$

Step 3.ii. Match coefficients to identify the unknown vector $\left[\alpha_{0}^{(n)} ; \alpha_{1}^{(n)} ; w c ; \psi_{w} ; v c ; \psi_{v} ; x c ; \psi_{x}\right]$.

In this context, we limit ourselves to comparing our approximation with the exact solution in the limit as $\rho \rightarrow 1$, in which the following closed-form solution is available: ${ }^{12}$

$$
\begin{aligned}
v c_{t}=\frac{\beta(\mu-v)}{1-\beta}+\psi_{v} \hat{p}_{t}, & \psi_{v}=\frac{1-\beta \rho_{p}-\sqrt{\left(1-\beta \rho_{p}\right)^{2}-2 \beta^{2} \phi_{p}^{2} \sigma^{2}\left(e^{(\gamma-1) \theta+\frac{(\gamma-1)^{2} \theta^{2} \delta^{2}}{2}}-1\right)}}{\beta(1-\gamma) \phi_{p}^{2} \sigma^{2}} \\
x c_{t}=\frac{\mu-v}{1-\beta}+\frac{1}{\beta} \psi_{v} \hat{p}_{t}, & v \doteq \frac{(\gamma-1) \sigma^{2}}{2}+\frac{(\gamma-1) \psi_{v}^{2} \phi_{p}^{2} \sigma^{2}}{2} p+\frac{e^{(\gamma-1) \theta+\frac{(\gamma-1)^{2} \theta^{2} \delta^{2}}{2}}-1}{\gamma-1} p
\end{aligned}
$$

The affine approximation recovers the exact solution, as

$$
\begin{aligned}
& w c_{t}=-\ln (1-\beta), \quad v c_{t}=\beta x c_{t}, \quad \sum_{n=0}^{\infty} e^{\alpha_{0}^{(n)}}=\frac{1}{1-\beta}, \quad \sum_{n=0}^{\infty} e^{\alpha_{0}^{(n)}} \alpha_{1}^{(n)}=0 \\
& \alpha_{0}^{(n)}=\alpha_{0}^{(n-1)}+\ln \left(\beta e^{(1-\gamma)(\mu+v c-x c)}\right)+\frac{(1-\gamma)^{2} \sigma^{2}}{2}+\left[\frac{\left[(1-\gamma) \psi_{v}+\alpha_{1}^{(n-1)}\right]^{2} \phi_{p}^{2} \sigma^{2}}{2}+e^{(\gamma-1) \theta+\frac{(\gamma-1)^{2} \theta^{2} \delta^{2}}{2}}-1\right] p \\
& \alpha_{1}^{(n)}=\alpha_{1}^{(n-1)} \rho_{p}+(1-\gamma)\left(\psi_{v} \rho_{p}-\psi_{x}\right)+\frac{\left[(1-\gamma) \psi_{v}+\alpha_{1}^{(n-1)}\right]^{2} \phi_{p}^{2} \sigma^{2}}{2}+e^{(\gamma-1) \theta+\frac{(\gamma-1)^{2} \theta^{2} \delta^{2}}{2}}-1
\end{aligned}
$$

has the same solution as (14), which implies $\alpha_{0}^{(n)}=n \ln (\beta)$ and $\alpha_{1}^{(n)}=0$.

\footnotetext{
${ }^{12}$ In the limit as $\rho \rightarrow 1$ we have $v_{t}=(1-\beta) c_{t}+\beta x_{t}$ and $x_{t}=\ln E_{t} e^{(1-\gamma) v_{t+1}} /(1-\gamma)$. It is easy to verify that an affine solution for $v_{t}-c_{t}$ in $p_{t}$ solves the problem exactly. The coefficient $\psi_{v}$ solves a quadratic equation, and we retain only the negative root as it is the only one that implies $\psi_{v}=0$ when disasters have no impact $-\theta=0$.
} 
It is straightforward to verify that we recover also the exact risk-free rate, when $\rho \rightarrow 1$, as

$$
r_{t}=-\ln \left(\beta e^{-\mu}\right)+\frac{(\gamma-1)^{2} \sigma^{2}}{2}-\frac{\gamma^{2} \sigma^{2}}{2}+\left(e^{(\gamma-1) \theta+\frac{(\gamma-1)^{2} \theta^{2} \delta^{2}}{2}}-e^{\gamma \theta+\frac{\gamma^{2} \theta^{2} \delta^{2}}{2}}\right) p_{t}
$$

\subsubsection{Perturbations around the deterministic steady state}

While our approximation recovers the exact solution as $\rho \rightarrow 1$, conventional perturbations do so only asymptotically. Even in the simple case with time-separable preferences $(\gamma=\rho)$, as $\rho \rightarrow 1$ a conventional $\ell$ th-order perturbation of the risk-free rate yields:

$$
r_{t}=-\ln \left(\beta e^{-\mu}\right)-\theta p_{t}-\sum_{j=1}^{\ell} \frac{\kappa_{j, t}}{j !} \stackrel{\ell \rightarrow \infty}{\rightarrow}-\ln \left(\beta e^{-\mu}\right)-\frac{\sigma^{2}}{2}-\left(e^{\theta+\theta^{2} \frac{\delta^{2}}{2}}-1\right) p_{t}
$$

where $\kappa_{j, t}$ is the $j$ th conditional cumulant of $\theta \varepsilon_{t+1}^{\xi}-\sigma \varepsilon_{t+1}^{c}$, hence $\sum_{j=1}^{\infty} \frac{\kappa_{j, t}}{j !}=\ln E_{t} e^{\theta \varepsilon_{t+1}^{\xi}-\sigma \varepsilon_{t+1}^{c}}$ by definition of the ccgf. Conventional perturbations yield the exact solution only as the order of approximation goes to infinity.

\subsubsection{Numerical example}

We calibrate the model using the values in Wachter (2013) reported in table 1. Figure 2 compares the exact solution to our affine approximation by plotting the map from the value of the state variable (disaster intensity) into the wealth-consumption ratio for different values of the elasticity of intertemporal substitution. We consider logutility, the standard value of 1.5 found in the long-run risk literature (e.g., Bansal and Yaron, 2004), and expected utility $(\rho=\gamma)$. The affine approximation recovers the exact solution at $\rho=1$ and remains extremely close to the global solution around the expansion point for specifications departing from logutility. The accuracy degrades only in tail regions of the state space.

\subsection{Portfolio choice in a small open economy}

We borrow the next example from Coeurdacier et al. (2011) as a case with an invalid local approximation around the deterministic steady state but a well-defined risky steady state. An affine approximation is also well-defined; in fact, section 4 shows the equivalence between our approximation and a risky steady state perturbation.

The representative consumer lives in a small open economy, receives an exogenous stream of income $Y_{t}=\theta$ for $t=0,1, \ldots$, and chooses a nonnegative consumption stream by investing an amount $A_{t}$ in foreign assets that pay off an exogenous interest rate $r$ with law of motion:

$$
r_{t+1}-r=\rho\left(r_{t}-r\right)+\sigma_{r} \varepsilon_{t+1}^{r}, \quad \varepsilon_{t}^{r} \sim \operatorname{Niid}(0,1)
$$

Investment is subject to a no-Ponzi condition. Markets are incomplete in that no other financial claim exists; the budget constraint is the backward-looking difference equation:

$$
C_{t}+A_{t} \leq Y_{t}+e^{r_{t}} A_{t-1}, \quad \lim _{h \rightarrow \infty}\left(\Pi_{s=1}^{h} e^{-r_{t+s}}\right) A_{t+h} \geq 0
$$


As required for a finite, stationary solution for consumption and asset holdings we assume $\beta e^{r}<1$ (see Chamberlain and Wilson, 2000).

Joint optimality of consumption and foreign-asset holdings implies:

$$
\begin{aligned}
0 & \geq \ln E_{t} e^{\ln (\beta)-\gamma \Delta c_{t+1}+r_{t+1}}, \quad=\text { if } A_{t}>0 \\
A_{t} & =e^{r_{t}} A_{t-1}+\theta-C_{t}=N_{t}-C_{t}
\end{aligned}
$$

where we define disposable resources $N_{t} \doteq e^{r_{t}} A_{t-1}+Y_{t}$ to serve as a state variable.

\subsubsection{Affine approximation}

We define variables $S_{t} \doteq \exp \left(N_{t}\right), X_{t} \doteq E_{t} R_{t+1}$, and $W_{t} \doteq \exp \left(R_{t}\right)$ to recast the problem in form (1) as:

$$
\begin{aligned}
0 & =\ln E_{t} e^{f\left(y_{t}, z_{t}, y_{t+1}, z_{t+1}\right)}, \quad f\left(y_{t}, z_{t}, y_{t+1}, z_{t+1}\right)=\left[\begin{array}{c}
\ln (\beta)-\gamma \Delta c_{t+1}+r_{t+1} \\
r_{t+1}-x_{t} \\
e^{r_{t}}-w_{t}
\end{array}\right] \\
z_{t+1} & =g\left(y_{t}, z_{t}\right)+\widetilde{\lambda}\left(y_{t}, z_{t}\right)\left(E_{t+1}-E_{t}\right) y_{t+1}+\sigma\left(z_{t}\right) \varepsilon_{t+1}^{r} \\
g\left(y_{t}, z_{t}\right) & =\left[\begin{array}{l}
\theta+\left(s_{t}-e^{c_{t}}\right) e^{x_{t}} \\
\left(1-\rho_{r}\right) r+\rho_{r} r_{t}
\end{array}\right], \quad \widetilde{\lambda}\left(y_{t}, z_{t}\right)=\left[\begin{array}{ccc}
0 & 0 & s_{t}-e^{c_{t}} \\
0 & 0 & 0
\end{array}\right], \quad \sigma\left(z_{t}\right)=\left[\begin{array}{c}
0 \\
\sigma_{r}
\end{array}\right]
\end{aligned}
$$

with decision variables $y_{t}=\left[c_{t} ; x_{t} ; w_{t}\right]$ and states $z_{t}=\left[s_{t} ; r_{t}\right]$.

Under a conjectured affine solution $y_{t}=y+\Psi\left(z_{t}-z\right)$ and definition $\lambda\left(z_{t}\right)=\widetilde{\lambda}\left[y+\Psi\left(z_{t}-z\right), z_{t}\right]$, the solution $[y, z, \Psi]$ can be found by solving matrix equation (5):

$$
\begin{aligned}
& 0=h+f_{3} y+f_{4} z+\widetilde{\mathcal{V}}(z) \\
& 0=g-z \\
& 0=f_{1} \Psi+f_{2}+\left(f_{3} \Psi+f_{4}\right)\left(g_{1} \Psi+g_{2}\right)+\widetilde{\mathcal{V}}_{1}(z)
\end{aligned}
$$

where:

$$
\begin{aligned}
& h=\left[\begin{array}{c}
\ln (\beta)+\gamma c \\
-x \\
e^{r}-w
\end{array}\right], f_{1}=\left[\begin{array}{ccc}
\gamma & 0 & 0 \\
0 & -1 & 0 \\
0 & 0 & -1
\end{array}\right], f_{2}=\left[\begin{array}{ll}
0 & 0 \\
0 & 0 \\
0 & e^{r}
\end{array}\right], f_{3}=\left[\begin{array}{ccc}
-\gamma & 0 & 0 \\
0 & 0 & 0 \\
0 & 0 & 0
\end{array}\right], f_{4}=\left[\begin{array}{ll}
0 & 1 \\
0 & 1 \\
0 & 0
\end{array}\right] \\
& g=\left[\begin{array}{c}
\theta+\left(s-e^{c}\right) e^{x} \\
r
\end{array}\right], g_{1}=\left[\begin{array}{ccc}
e^{x} & \left(s-e^{c}\right) e^{x} & 0 \\
0 & 0 & 0
\end{array}\right], g_{2}=\left[\begin{array}{ll}
0 & 0 \\
0 & \rho_{r}
\end{array}\right]
\end{aligned}
$$

and $\widetilde{\mathcal{V}}\left(z_{t}\right)=\frac{1}{2} \operatorname{diag}\left(\left(f_{3} \Psi+f_{4}\right)\left[I-\lambda\left(z_{t}\right) \Psi\right]^{-1} \sigma\left(z_{t}\right) \sigma\left(z_{t}\right)^{\prime}\left[I-\lambda\left(z_{t}\right) \Psi\right]^{-1 \prime}\left(f_{3} \Psi+f_{4}\right)^{\prime}\right)$.

Importantly, the derivative of entropy with respect to the endogenous state $s_{t}$ is nonzero. By arguments made clear in section 4 , a nontrivial matrix $\widetilde{\mathcal{V}}_{1}(z)$ affects the dynamics of the approximate system and can provide determinacy. Indeed, one can show that the affine approximation results in saddle-point stable dynamics for a wide range of values of the deep parameters. Coeurdacier et al. (2011) discuss the stationarity of the model around the risky steady state, so our stability result is not surprising once we realize the equivalence of affine approximations and first-order perturbations 
around the risky steady state. We leave to section 4 the proof of this proposition and hence the formal justification of affine methods as well as the characterization of saddle-path conditions.

\subsubsection{Perturbations around the deterministic steady state}

In this example, conventional perturbations around the deterministic steady state are not welldefined. In fact, in this example the deterministic steady state is the corner solution $C=0$ and $A=-\frac{\theta}{e^{r}-1}$, and perturbations around that point that associate with $C<0$ would be inadmissible.

We also rule out an interior solution, as it would imply a deterministic steady state with $\beta e^{r}=1$, a contradiction. (Had we assumed $\beta e^{r}=1$, then the pair $(c, a)$ would be indeterminate-any $A>-\frac{\theta}{e^{r}-1}$ would be a stationary value. Accordingly, we would have unit-root local dynamics that invalidate the local approximation.)

\section{Approximation method: Formal statement}

Proposition 1 provides the mathematical foundation for affine approximations. Part a. characterizes saddle-point stability of any solution of the nonlinear equations (5), adapting Blanchard and Kahn (1980) conditions to our context. Relative to conventional linearizations, the determinacy of equilibrium dynamics is affected by the evaluation of derivatives at $\left[y_{t}, z_{t}\right]=[y, z]$ and by the presence of the dynamic entropy component. Part $b$. shows how affine approximations coincide with linear perturbations around the risky steady state, thereby inheriting their formal justification based on the implicit function and Taylor theorems.

\subsection{Formal derivation}

To set the ground for perturbations, we consider the parametrized family of system (1):

$$
\begin{aligned}
0 & =E_{t} x_{t+1}^{q}+\tau \mathcal{V}_{t}\left[\exp \left(x_{t+1}^{q}\right)\right]+(1-\tau) \widetilde{\mathcal{V}}\left(z_{t}^{q}, q\right) \\
z_{t+1}^{q} & =g\left[y\left(z_{t}^{q}, q, \tau\right), z_{t}^{q}\right]+\lambda\left(z_{t}^{q}\right)\left(E_{t+1}-E_{t}\right) y\left[z\left(z_{t}^{q}, q, \varepsilon_{t+1}, \tau\right), q, \tau\right]+\sigma\left(z_{t}^{q}\right) q \varepsilon_{t+1} \\
x_{t+1}^{q} & \doteq h\left[y\left(z_{t}^{q}, q\right), z_{t}^{q}, \tau\right]+f_{3} y\left[z\left(z_{t}^{q}, q, \varepsilon_{t+1}, \tau\right), q, \tau\right]+f_{4} z\left(z_{t}^{q}, q, \varepsilon_{t+1}, \tau\right)
\end{aligned}
$$

We are looking for solutions for jump and state variables $y_{t}^{q}=y\left(z_{t}^{q}, q, \tau\right)$ and $z_{t+1}^{q}=z\left(z_{t}^{q}, q, \varepsilon_{t+1}, \tau\right)$, where scalar $q$ denotes the amount of risk in the economy, and scalar $\tau$ indicates whether entropy is evaluated using the true policy function or using a function $y_{t}^{q}=\widetilde{y}^{q}+\widetilde{\Psi}^{q}\left(z_{t}^{q}-\widetilde{z}^{q}\right)$ for some coefficients $\widetilde{y}^{q}, \widetilde{z}^{q}$, and $\widetilde{\Psi}^{q}$. Under $q=\tau=1$ the dynamics coincide with the original model (1). Entropy $w\left(z_{t}^{q}, q, \tau\right) \doteq \mathcal{V}_{t}\left[\exp \left(x_{t+1}^{q}\right)\right]$ is assumed to be differentiable in $z_{t}^{q}$ for all $q \in[0,1]$.

In this context, we also define an affine approximation with coefficients $y^{q}, z^{q}$, and $\Psi^{q}$ that solves

$$
\begin{aligned}
& 0=h\left(y^{q}, z^{q}\right)+f_{3} y^{q}+f_{4} z^{q}+\widetilde{\mathcal{V}}\left(z^{q}, q\right), \quad z^{q}=g\left(y^{q}, z^{q}\right) \\
& 0=f_{1}^{q} \Psi^{q}+f_{2}^{q}+\left(f_{3} \Psi^{q}+f_{4}\right)\left(g_{1}^{q} \Psi^{q}+g_{2}^{q}\right)+\widetilde{\mathcal{V}}_{1}\left(z^{q}, q\right)
\end{aligned}
$$

where $f_{i}^{q} \doteq h_{i}\left(y^{q}, z^{q}\right)$ and $g_{i}^{q} \doteq g_{i}\left(y^{q}, z^{q}\right)$, with $\widetilde{\mathcal{V}}\left(z^{q}, q\right) \doteq \kappa\left[\left(f_{3} \Psi^{q}+f_{4}\right)\left(I-\lambda\left(z^{q}\right) \Psi^{q}\right)^{-1} \sigma\left(z^{q}\right) q ; z^{q}\right]$ a differentiable function for all $q \in[0,1]$. At $q=1$, we recover (5) and $y^{q}=y, z^{q}=z$ and $\Psi^{q}=\Psi$. 
Definition. A risky steady state (RSS) of system (16) is a point $z_{t}^{q}=\widetilde{z}^{q}$ and $\tau=1$ such that $\widetilde{z}^{q}=z\left(z^{q}, q, 0,1\right)$ and $\widetilde{y}^{q}=y\left(z^{q}, q, 1\right)$, i.e., a point where agents choose to stay $i$ ) while expecting shocks ex ante; $i$ ) when ex-post realized shocks are zero; and iii) while forming expectations consistent with the exact solution.

The risky steady state is a better expansion point than the deterministic steady state because it implies no approximation around $q=0$. But note that the definition of risky steady state relies on a specific description of how people form expectations over future states of nature. Whenever the exact solution is unknown to the modeler, however, the calculation of the risky steady is unfeasible. A more useful definition is that of a feasible risky steady state.

Definition. A feasible risky steady state (FRSS) of system (16) is a point $z_{t}^{q}=\widetilde{z}^{q}$ and $\tau=0$ such that $\widetilde{z}^{q}=z\left(\widetilde{z}^{q}, q, 0,0\right)$ and $\widetilde{y}^{q}=y\left(\widetilde{z}^{q}, q, 0\right)$, i.e., a point where agents choose to stay $i$ ) while expecting shocks ex ante; $i$ ) when ex-post realized shocks are zero; and iii) while forming expectations consistent with a linear approximation of the solution around the FRSS. ${ }^{13}$

A FRSS $\left(z_{t}^{q}, \tau\right)=\left(z^{q}, 0\right)$ is called a saddle point if matrices

$$
\Gamma \doteq\left[\begin{array}{cc}
f_{4} & f_{3} \\
I_{n_{z}} & 0
\end{array}\right] \in \mathbb{C}^{n_{y}+n_{z} \times n_{y}+n_{z}}, \quad \Xi^{q} \doteq\left[\begin{array}{cc}
-f_{2}\left(\widetilde{y}^{q}, \widetilde{z}^{q}\right)-\widetilde{\mathcal{V}}_{1}\left(\widetilde{z}^{q}, q\right) & -f_{1}\left(\widetilde{y}^{q}, \widetilde{z}^{q}\right) \\
g_{2}\left(\widetilde{y}^{q}, \widetilde{z}^{q}\right) & g_{1}\left(\widetilde{y}^{q}, \widetilde{z}^{q}\right)
\end{array}\right] \in \mathbb{C}^{n_{y}+n_{z} \times n_{y}+n_{z}}
$$

have $n_{z}$ generalized eigenvalues $\alpha\left(\Gamma, \Xi^{q}\right) \doteq\left\{\alpha \in \mathbb{C}: \operatorname{det}\left(\Gamma \alpha-\Xi^{q}\right)=0\right\}$ inside the unit circle and $n_{y}$ outside. A first-order approximation around a saddle point is a saddle path.

Proposition 1. (a) A solution $\left(y^{q}, z^{q}, \Psi^{q}\right)$ of system (17) (and (5) at $q=1$ ) has unique and bounded dynamics if and only if the associated matrices $\Gamma$ and $\Xi^{q}$ have $n_{z}$ generalized eigenvalues inside the unit circle and $n_{y}$ outside.

(b) Suppose that a FRSS $\left(z_{t}^{q}, \tau\right)=\left(z^{q}, 0\right)$ is a saddle point. Then, i) functions $y_{t}^{q}=y\left(z_{t}^{q}, q, \tau\right)$ and $z_{t+1}^{q}=z\left(z_{t}^{q}, q, \varepsilon_{t+1}, \tau\right)$ are unique and differentiable in a neighborhood of the FRSS; and ii) the affine approximate solution $\left(y^{q}, z^{q}, \Psi^{q}\right)$ of system (17) coincides with the coefficients from a linear perturbation around the FRSS of system (16): $y^{q}=\widetilde{y}^{q}, z^{q}=\widetilde{z}^{q}, \Psi^{q}=y_{1}\left(\widetilde{z}^{q}, q, 0\right)$.

Appendices B and C provide a proof of the proposition.

\subsection{Relationship with conventional perturbations}

Our affine approximation (and hence a first-order perturbation around the risky steady state, by proposition 1) is not nested in conventional perturbations around the deterministic steady state $\left(z_{t}, q\right)=(\bar{z}, 0)$ (e.g., à la Schmitt-Grohé and Uribe, 2004). ${ }^{14}$ Proposition 2 provides sufficient

\footnotetext{
${ }^{13}$ Note that this is precisely the definition in Coeurdacier et al. (2011), who conjecture a linear solution and plug it into the nonlinear equation. Note also that Coeurdacier et al. rely on an additional second-order approximation to solve for the perturbation coefficients, and in so doing they effectively replace entropy with variance-an unnecessary ad-hoc step. The online appendix elaborates this point.

${ }^{14}$ The online appendix discusses this further by comparing analytically the generalized affine approximation and a third-order perturbation around the deterministic steady state.
} 
conditions under which nesting does not occur and takes stock of the lessons from the examples of section 3.

Note that one can at most reconstruct the implicit functions $y(\bar{z}, q)$ and $y_{1}(\bar{z}, q)$ using output from $\ell$ th-order perturbations around the deterministic steady state $\left(z_{t}, q\right)=(\bar{z}, 0)$ as:

$$
y(\bar{z}, q)=\left.\lim _{\ell \rightarrow \infty} \sum_{i=1}^{\ell} \frac{1}{i !} \frac{\partial^{i} y(\bar{z}, q)}{\partial q^{i}}\right|_{q=0} q^{i}, \quad y_{1}(\bar{z}, q)=\left.\lim _{\ell \rightarrow \infty} \sum_{i=0}^{\ell} \frac{1}{i !} \frac{\partial^{i} y_{1}(\bar{z}, q)}{\partial q^{i}}\right|_{q=0} q^{i}
$$

as long as the implicit functions $y(\bar{z}, q)$ and $y_{1}(\bar{z}, q)$ have convergent Taylor series at $q=0$ with a sufficiently large radius of convergence. In this context, the implicit functions of interest are $y(z, 1)$ and $y_{1}(z, 1)$, so a necessary (but not sufficient) condition for nesting is that the radius of convergence of the Taylor series is larger than unity. Therefore:

Proposition 2. If $y(z, 1) \neq y(\bar{z}, 1)$ or $y_{1}(z, 1) \neq y_{1}(\bar{z}, 1)$, then risky steady state perturbations are not nested in deterministic steady state perturbations of arbitrary order $\ell$.

Proposition 2 provides sufficient conditions for the absence of nesting. Relevant examples where these conditions are satisfied include the small open economy described in section 3 as well as the production economy of section 6. Moreover, even when nesting is possible, the speed of convergence as the order of approximation $\ell$ increases can be impractically large. For example, in the model of Campbell and Cochrane (1999), figures 1 and 2 show how third-order perturbations fall short of providing a sufficiently accurate approximation of the risky steady state perturbation.

\section{Approximate equilibrium risk pricing}

This section inspects further the general approximate solution formula (5) applied specifically to the pricing of assets. ${ }^{15}$ After having solved for the equilibrium allocation, we can price assets in zero net supply by relying on the no-arbitrage relation, $0=\ln E_{t}^{\mathbb{P}} e^{m_{t+1}+r_{t+1}^{j}}$, where $m$ is the stochastic discount factor and $r^{j}$ the return paid off by the $j$ th claim; the expectations operator makes explicit its reference to the physical probability measure $(\mathbb{P})$. While these pricing implications can be included in system (1), we illustrate them separately without loss of generality to make clear how affine approximations help inspecting equilibrium risk prices.

The approximate equilibrium allocation using the method of section 2 includes a state vector $\hat{z}_{t} \doteq z_{t}-z \in \mathbb{R}^{n_{z}}$ and a $\log$ cashflow process $d_{t}=a_{t}+S_{d}^{\prime} y_{t} \in \mathbb{R}$, with $a_{t}$ a trend component with dynamics $a_{t+1}=\mu_{d}+a_{t}+C_{a} \hat{z}_{t}+D_{a}\left(\hat{z}_{t}\right) \varepsilon_{t+1}$ and $S_{d} \in \mathbb{R}^{n_{y}}$ a selection matrix from the vector of jump variables $y_{t} \in \mathbb{R}^{n_{y}}$, whose approximate joint distribution under the physical probability is:

$$
\left[\begin{array}{c}
\hat{z}_{t+1} \\
\Delta d_{t+1}
\end{array}\right]=\left[\begin{array}{c}
0 \\
\mu_{d}
\end{array}\right]+\left[\begin{array}{l}
A \\
C
\end{array}\right] \hat{z}_{t}+\left[\begin{array}{c}
B\left(\hat{z}_{t}\right) \\
D\left(\hat{z}_{t}\right)
\end{array}\right] \varepsilon_{t+1}, \quad \kappa\left[\alpha\left(z_{t}\right) ; z_{t}\right] \doteq \ln E_{t}^{\mathbb{P}}\left[e^{\alpha\left(z_{t}\right)^{\prime} \varepsilon_{t+1}}\right], \alpha: \mathbb{R}^{n_{z}} \rightarrow \mathbb{R}^{n_{\varepsilon}}
$$

with matrices $A \doteq g_{1} \Psi+g_{2}, C \doteq C_{a}-S_{d}^{\prime} \Psi\left(I_{n_{z}}-A\right), B\left(\hat{z}_{t}\right) \doteq\left(I_{n_{z}}-\lambda\left(z_{t}\right) \Psi\right)^{-1} \sigma\left(z_{t}\right)$ and $D\left(\hat{z}_{t}\right) \doteq$ $D_{a}\left(\hat{z}_{t}\right)+S_{d}^{\prime} \Psi\left(I_{n_{z}}-\lambda\left(z_{t}\right) \Psi\right)^{-1} \sigma\left(z_{t}\right)$.

\footnotetext{
${ }^{15}$ This includes welfare costs of economic fluctuations, as they are hold-to-maturity risk premia at the margin (Alvarez and Jermann, 2004; Lopez, 2014).
} 
The equilibrium $\log$ risk-free rate is described by the Euler equation, $0=\ln E_{t}^{\mathbb{P}} e^{m_{t+1}+r_{t}}$, where the one-period $\log$ stochastic discount factor $m_{t}$ is a linear transformation of the vector of jump, state and trend variables such that $\left(E_{t+1}-E_{t}\right) m_{t+1}=-\gamma\left(\hat{z}_{t}\right)^{\prime} \varepsilon_{t+1}$, with the price of risk $\gamma\left(\hat{z}_{t}\right) \in \mathbb{R}^{n_{\varepsilon}}$. It follows that the stochastic discount factor has form:

$$
m_{t+1}=-r\left(\hat{z}_{t}\right)-\kappa\left[-\gamma\left(\hat{z}_{t}\right)^{\prime} ; z_{t}\right]-\gamma\left(\hat{z}_{t}\right)^{\prime} \varepsilon_{t+1}
$$

\subsection{Asset pricing formulas}

Under structure (18) and (19), we are able to characterize in proposition 3 the dependence of generalized equilibrium term-structure components on the states. By proposition 1, proposition 3 also describes equilibrium cashflow strip yields linearized around the risky steady state.

Proposition 3. Under assumptions (18) and (19), we obtain the following linear approximations of yields and risk premia:

(a) The nth cashflow strip yield, $y_{d, t}^{(n)} \doteq-\frac{1}{n} \ln \left(P_{d, t}^{(n)} / D_{t}\right)$, with $P_{d, t}^{(n)}=E_{t}^{\mathbb{P}}\left(M_{t, t+n} D_{t+n}\right)$ the no-arbitrage price of the nth strip of cashflow process $d$, has the approximate affine form

$$
y_{d, t}^{(n)}=-\frac{1}{n} A^{(n)}-\frac{1}{n} B_{z}^{(n)} \hat{z}_{t}
$$

with term structure coefficients determined by the matrix difference equations

$$
\begin{aligned}
A^{(n)}= & A^{(n-1)}+\mu_{d}-r(0)-\kappa\left[-\gamma(0)^{\prime} ; z\right]+\kappa\left[-\gamma(0)^{\prime}+V_{n-1}(0)^{\prime} ; z\right] \\
B_{z}^{(n)}= & B_{z}^{(n-1)} A+C-r_{1}(0)-\kappa_{2}\left[-\gamma(0)^{\prime} ; z\right]+\kappa_{2}\left[-\gamma(0)^{\prime}+V_{n-1}(0)^{\prime} ; z\right] \\
& +\kappa_{1}\left[-\gamma(0)^{\prime} ; z\right] \gamma_{1}(0)+\kappa_{1}\left[-\gamma(0)^{\prime}+V_{n-1}(0)^{\prime} ; z\right]\left[-\gamma_{1}(0)+V_{1, n-1}(0)\right]
\end{aligned}
$$

with boundary condition $\left[A^{(0)} ; B_{z}^{(0)}\right]=0$, where $V_{n}\left(\hat{z}_{t}\right)^{\prime} \doteq D\left(\hat{z}_{t}\right)+B_{z}^{(n)} B\left(\hat{z}_{t}\right)$ controls the loading of the unexpected component of the nth holding period log return on the shock.

(b) The holding-period risk premium $r_{d, t+1}^{e,(n)} \doteq p_{0, t}^{(1)}+p_{d, t+1}^{(n-1)}-p_{d, t}^{(n)}$ commanded by the n-period ahead cashflow strip is:

$$
\ln E_{t}^{\mathbb{P}} R_{d, t+1}^{e,(n)}=\kappa\left[-\gamma\left(\hat{z}_{t}\right)^{\prime} ; z_{t}\right]+\kappa\left[V_{n-1}\left(\hat{z}_{t}\right)^{\prime} ; z_{t}\right]-\kappa\left[-\gamma\left(\hat{z}_{t}\right)^{\prime}+V_{n-1}\left(\hat{z}_{t}\right)^{\prime} ; z_{t}\right]
$$

which coincides with negative coentropy $-C_{t}\left(M_{t+1}, P_{d, t+1}^{(n-1)}\right)$ under stochastic discount factor (19) and the approximate cashflow strip price. ${ }^{16}$ This result implies:

$$
r_{d, t+1}^{e,(n)}=\ln E_{t}^{\mathbb{P}} R_{d, t+1}^{e,(n)}-\kappa\left[V_{n-1}\left(\hat{z}_{t}\right)^{\prime} ; z_{t}\right]+V_{n-1}\left(\hat{z}_{t}\right)^{\prime} \varepsilon_{t+1}
$$

\footnotetext{
${ }^{16}$ Conditional coentropy of two random variables can be defined as $C_{t}\left(e^{x_{t+1}}, e^{y_{t+1}}\right) \doteq \mathcal{V}_{t}\left(e^{x_{t+1}+y_{t+1}}\right)-\mathcal{V}_{t}\left(e^{x_{t+1}}\right)-\mathcal{V}_{t}\left(e^{y_{t+1}}\right)$ (see also Hansen, 2012; Backus et al., 2018).
} 
(c) The per-period hold-to-maturity risk premium commanded by the nth cashflow strip is

$$
\frac{1}{n} \ln E_{t}^{\mathbb{P}}\left(\frac{P_{0, t}^{(n)} D_{t+n}}{P_{d, t}^{(n)}}\right)=\frac{1}{n}\left[A_{g}^{(n)}+A_{0}^{(n)}-A_{d}^{(n)}\right]+\frac{1}{n}\left[B_{g, z}^{(n)}+B_{0, z}^{(n)}-B_{d, z}^{(n)}\right] \hat{z}_{t}
$$

where subscripts 0 and $d$ index the term structure coefficients associated with real bonds and the relevant cashflow process respectively, and where coefficients $\left\{A_{g}^{(n)}, B_{g}^{(n)}\right\}$ determine the term structure of anticipated cashflow growth $\frac{1}{n} \ln E_{t}^{\mathbb{P}}\left(\frac{D_{t+n}}{D_{t}}\right)=\frac{1}{n} A_{g}^{(n)}+\frac{1}{n} B_{g}^{(n)} \hat{z}_{t}$ :

$$
\begin{aligned}
& A_{g}^{(n)}=\mu_{d}+A_{g}^{(n-1)}+\kappa\left[W_{n-1}(0)^{\prime} ; z\right] \\
& B_{g}^{(n)}=B_{g}^{(n)} A+C+\kappa_{1}\left[W_{n-1}(0)^{\prime} ; z\right] W_{1, n-1}(0)+\kappa_{2}\left[W_{n-1}(0)^{\prime} ; z\right]
\end{aligned}
$$

with boundary condition $\left[A_{g}^{(0)} ; B_{g}^{(0)}\right]=0$, where $W_{n}\left(\hat{z}_{t}\right)^{\prime} \doteq D\left(\hat{z}_{t}\right)+B_{g}^{(n)} B\left(\hat{z}_{t}\right)$ controls the loading of the unexpected component of n-period ahead cashflow growth on the shock.

Appendix D provides a proof of the proposition.

The approximate equilibrium prices of strips characterized by proposition 3 are the basis to price other payoffs. For example, given equilibrium strip prices, the log price-dividend ratio and return on the market portfolio can be constructed as:

$$
\ln \left(\frac{P_{t}}{D_{t}}\right)=\ln \left(\sum_{n=1}^{\infty} e^{-n y_{d, t}^{(n)}}\right), \quad \quad E_{t}^{\mathbb{P}} R_{t+1}^{m}=\sum_{n=1}^{\infty} \omega_{n, t} E_{t}^{\mathbb{P}} R_{d, t+1}^{(n)}
$$

where $\omega_{n, t} \doteq e^{-n y_{d, t}^{(n)}} / \sum_{n=1}^{\infty} e^{-n y_{d, t}^{(n)}}$ with $\sum_{n=1}^{\infty} \omega_{n, t}=1$. The approximate distribution of the portfolio can be constructed using simulated moments of strip prices and returns.

More generally, we can characterize the approximate $\mathbb{Q}$-distribution of the state vector and cashflows and how it distorts the $\mathbb{P}$-distribution, as shown in proposition 4.

Proposition 4. The vector process $[z ; \Delta d]$ has the approximate ccgf under the physical $(\mathbb{P})$ and risk-neutral $(\mathbb{Q})$ probability measures:

$$
\begin{aligned}
& \ln E_{t}^{\mathbb{P}}\left[e^{u_{z}^{\prime} \hat{z}_{t+1}+u_{d} \Delta d_{t+1}}\right]=u^{\prime} \mu+\kappa\left[u^{\prime} \Sigma\left(\hat{z}_{t}\right) ; z_{t}\right]+u^{\prime} \Phi \hat{z}_{t} \\
& \ln E_{t}^{\mathbb{Q}}\left[e^{u^{\prime} \hat{z}_{t+1}+u_{d} \Delta d_{t+1}}\right]=\ln E_{t}^{\mathbb{P}}\left[e^{u_{z}^{\prime} \hat{z}_{t+1}+u_{d} \Delta d_{t+1}}\right]+\kappa\left[-\gamma\left(\hat{z}_{t}\right)^{\prime}+u^{\prime} \Sigma\left(\hat{z}_{t}\right) ; z_{t}\right]-\kappa\left[-\gamma\left(\hat{z}_{t}\right)^{\prime} ; z_{t}\right]-\kappa\left[u^{\prime} \Sigma\left(\hat{z}_{t}\right) ; z_{t}\right]
\end{aligned}
$$

for $u=\left[u_{z} ; u_{d}\right] \in \mathbb{R}^{n_{z}+1}$, where $\mu \doteq\left[0_{n_{z}} ; \mu_{d}\right] \in \mathbb{R}^{n_{z}+1}, \Phi \doteq[A ; C] \in \mathbb{R}^{n_{z}+1 \times n_{z}}$ and $\Sigma\left(\hat{z}_{t}\right) \doteq$ $\left[B\left(\hat{z}_{t}\right) ; D\left(\hat{z}_{t}\right)\right] \in \mathbb{R}^{n_{z}+1 \times n_{\varepsilon}}$.

Appendix E proves this proposition.

\subsection{Asset pricing diagnostics}

The literature provides a set of diagnostic tools that can be used to test a model of the stochastic discount factor. An important criterion to evaluate the accuracy of an approximation method is that it correctly captures these implications. These diagnostic properties make clear how an approximation 
method that correctly captures the term structures of equities and bonds, and especially their long-run properties, is a method that correctly captures this decomposition of pricing kernels and, more specifically, investors' marginal utility. Our emphasis on evaluating the quality of the approximation via the term structures of claims to different cashflow processes in section 6 rests on this observation.

A first diagnostic tool to assess a model of the discount factor is the Hansen and Jagannathan (1991) bound, which shows how no-arbitrage pricing implies that the volatility of the discount factor must dominate empirical measures of the maximal risk-return tradeoff. Our affine approximation provides a simple expression for the bound:

$$
\left|\frac{E_{t}^{\mathbb{P}} R_{t+1}^{e}}{s t d_{t}^{\mathbb{P}}\left(R_{t+1}^{e}\right)}\right| \leq \frac{s t d_{t}^{\mathbb{P}}\left(M_{t+1}\right)}{E_{t}^{\mathbb{P}} M_{t+1}}=\frac{\sqrt{E_{t}^{\mathbb{P}} e^{2 m_{t+1}}-\left(E_{t}^{\mathbb{P}} e^{m_{t+1}}\right)^{2}}}{E_{t}^{\mathbb{P}} M_{t+1}} \approx \sqrt{e^{\kappa\left[-2 \gamma\left(\hat{z}_{t}\right) ; z_{t}\right]-2 \kappa\left[-\gamma\left(\hat{z}_{t}\right) ; z_{t}\right]}-1}
$$

for all available excess returns. Similar simple expressions for Backus et al. (2014) entropy-based bounds are straightforward to derive.

Our affine approximation also provides tractable expressions for the decompositions of Alvarez and Jermann (2005) and Hansen and Scheinkman (2009). They show how, under appropriate regularity conditions, the stochastic discount factor can be decomposed as $M_{t+1}=M_{t+1}^{P} M_{t+1}^{T}$, where a transient component $M_{t+1}^{T}$ controls the pricing of long-duration bonds and a martingale component $M_{t+1}^{P}$ with $E_{t}^{\mathbb{P}} M_{t+1}^{P}=1$ controls the maximum risk premium in the complete-market economy. Two main properties of the decomposition that rest on the no-arbitrage pricing formula and Jensen's inequality are (i) the relationship between the transient component of the discount factor and the holding-period return on a infinite-maturity zero-coupon bond,

$$
m_{t+1}^{T}=-\lim _{n \rightarrow \infty} r_{0, t+1}^{(n)}
$$

and (ii) the property of the entropy ratio,

$$
\frac{\mathcal{V}_{t}^{\mathbb{P}}\left(M_{t+1}^{P}\right)}{\mathcal{V}_{t}^{\mathbb{P}}\left(M_{t+1}\right)}=1-\frac{E_{t}^{\mathbb{P}} r_{0, t+1}^{e,(\infty)}}{\mathcal{V}_{t}^{\mathbb{P}}\left(M_{t+1}\right)} \geq 1-\frac{E_{t}^{\mathbb{P}} r_{0, t+1}^{e,(\infty)}}{\max E_{t}^{\mathbb{P}} r_{t+1}^{e}}
$$

where the maximum is taken over all available excess returns.

Proposition 5 constructs the approximate decomposition, which Hansen and Scheinkman (2009) show it can be understood using the solution $[\delta ; f]$ to the eigenfunction problem:

$$
E_{t}^{\mathbb{P}}\left[M_{t+1} f\left(\hat{z}_{t+1}\right)\right]=\delta f\left(\hat{z}_{t}\right)
$$

for some function $f: \mathbb{R}^{n+1} \rightarrow \mathbb{R}$ and scalar $\delta \in \mathbb{R}$, with transient and martingale components constructed as $M_{t+1}^{T}=\delta f\left(\hat{z}_{t}\right) / f\left(\hat{z}_{t+1}\right)$ and $M_{t+1}^{P}=M_{t+1} f\left(\hat{z}_{t+1}\right) / \delta f\left(\hat{z}_{t}\right)$.

Proposition 5. In the context of the general model of section 2, the approximate generalized affine 
solution of the eigenfunction problem is $\delta \in \mathbb{R}$ and $f\left(\hat{z}_{t}\right)=e^{u_{z}^{\prime} \hat{z}_{t}}$ with:

$$
\begin{aligned}
0= & \ln (\delta)-r(0)+\kappa\left[-\gamma(0)^{\prime}+V(0)^{\prime} ; z\right]-\kappa\left[-\gamma(0)^{\prime} ; z\right] \\
u_{z}^{\prime}= & u_{z}^{\prime} A \hat{z}_{t}-r_{1}(0) \hat{z}_{t}-\kappa_{2}[-\gamma(0) ; z]+\kappa_{2}[-\gamma(0)+V(0) ; z] \\
& +\kappa_{1}\left[\gamma(0)^{\prime} ; z\right] \gamma_{1}(0)+\kappa_{1}\left[-\gamma(0)^{\prime}+V(0)^{\prime} ; z\right]\left[-\gamma(0)^{\prime}+V(0)^{\prime}\right]
\end{aligned}
$$

where $V\left(\hat{z}_{t}\right)^{\prime} \doteq u_{z}^{\prime} B\left(\hat{z}_{t}\right)$.

Appendix F provides a proof of the proposition. Appendix G shows the equivalence of HansenScheinkman and Alvarez-Jermann decompositions in this context of (approximate) affine pricing.

\subsection{Inspecting the mechanism}

Affine approximations are particularly useful to provide an intuitive understanding of the macroeconomic forces that drive the prices of financial claims and the risk premia they command. Using equation $r_{t+1}^{e,(n)}=E_{t}^{\mathbb{P}} r_{t+1}^{e,(n)}+V_{n-1}\left(\hat{z}_{t}\right)^{\prime} \varepsilon_{t+1}$ from proposition 3, we can attribute the coentropy of the quantity of risk in the $n$th strip return with a shock along direction $\alpha\left(\hat{z}_{t}\right)$ to two components,

$$
-C_{t}^{\mathbb{P}}\left(\exp \left[\alpha\left(\hat{z}_{t}\right)^{\prime} \varepsilon_{t+1}\right], \exp \left[(\underbrace{D\left(\hat{z}_{t}\right)}_{\begin{array}{c}
\text { short-run } \\
\text { cashflow risk }
\end{array}}+\underbrace{B_{z}^{(n-1)} B\left(\hat{z}_{t}\right)}_{\begin{array}{c}
\text { long-run cashflow } \\
\text { and discount-rate risk }
\end{array}}) \varepsilon_{t+1}\right]\right)
$$

which are the basis to understand the shape of the term structure of holding-period risk premia when $\alpha\left(\hat{z}_{t}\right)=-\gamma\left(\hat{z}_{t}\right)$. The first element on the right-hand side of the equation controls the cashflow effect due to contemporaneous shocks to dividends. The second element captures income and substitution effects of past shocks as well as the role of each state variable in shaping the term structure.

Another way the generalized affine approximation facilitates an intuitive understanding of the asset-pricing implications of the macro-finance model is by providing simple expressions for the dynamic value decomposition proposed by Borovicka and Hansen (2014) as measures to quantify the exposures of cashflows to shocks over alternative horizons and the corresponding compensations commanded by investors. In particular, for any marginal increase in one-step ahead uncertainty along dimension $\alpha\left(\hat{z}_{t}\right)$ we can define cashflow and discount-rate elasticities as:

$$
\begin{aligned}
\varepsilon_{g, t}^{(n)} & \doteq \frac{d}{d r} \ln E_{t}^{\mathbb{P}}\left[\frac{D_{t+n}}{D_{t}} e^{r \alpha\left(\hat{z}_{t}\right)^{\prime} \varepsilon_{t+1}-\kappa\left[r \alpha\left(\hat{z}_{t}\right)^{\prime} ; z_{t}\right]}\right]_{r=0}=\kappa_{1}\left[D\left(\hat{z}_{t}\right)+B_{g}^{(n-1)} B\left(\hat{z}_{t}\right) ; z_{t}\right] \alpha\left(\hat{z}_{t}\right) \\
\varepsilon_{p, t}^{(n)} & \doteq \frac{d}{d r} \ln E_{t}^{\mathbb{P}}\left[\frac{D_{t+n}}{D_{t}} e^{r \alpha\left(\hat{z}_{t}\right)^{\prime} \varepsilon_{t+1}-\kappa\left[r \alpha\left(\hat{z}_{t}\right)^{\prime} ; z_{t}\right]}\right]_{r=0}-\frac{d}{d r} \ln E_{t}^{\mathbb{P}}\left[M_{t, t+n} \frac{D_{t+n}}{D_{t}} e^{r \alpha\left(\hat{z}_{t}\right)^{\prime} \varepsilon_{t+1}-\kappa\left[r \alpha\left(\hat{z}_{t}\right)^{\prime} ; z_{t}\right]}\right]_{r=0} \\
& =\kappa_{1}\left[D\left(\hat{z}_{t}\right)+B_{g}^{(n-1)} B\left(\hat{z}_{t}\right) ; z_{t}\right] \alpha\left(\hat{z}_{t}\right)-\kappa_{1}\left[-\gamma\left(\hat{z}_{t}\right)+D\left(\hat{z}_{t}\right)+B_{z}^{(n-1)} B\left(\hat{z}_{t}\right) ; z_{t}\right] \alpha\left(\hat{z}_{t}\right)
\end{aligned}
$$

Appendix G derives expression (22). These elasticities capture the impact of current shocks on future cashflows $\left(\varepsilon_{g, t}^{(n)}\right)$ and on future expected returns $\left(\varepsilon_{p, t}^{(n)}\right)$, while the impact on valuations can be recovered as the value elasticity $\varepsilon_{g, t}^{(n)}-\varepsilon_{p, t}^{(n)}$. 


\section{Applications}

This section illustrates the performance of our generalized affine approximation in terms of risk pricing at different horizons, and by applying it to a more challenging model—a production economy. Models with Campbell-Cochrane habits are particularly suited to test our approximation as they display strong heteroskedasticity; the state of the economy is driven by consumption news, which are endogenous objects outside an endowment economy. Models with time-varying disaster risk similarly produces variation in risk premia, while non-Gaussianities make loglinear-lognormal methods inapplicable.

We evaluate the quality of our approximation by comparing the term structures of zero-coupon claims and, in production economies, multiperiod Euler equation errors. This exercise decomposes the quality of the approximation at different time horizons, and for claims that are the basis for pricing other more complex assets and to characterize the investors' marginal utility. We define errors in the $n$-period Euler equation from a solution for consumption $c^{(0)}\left(z_{t}\right)$ as:

$$
\operatorname{EEE}^{(n)}\left(z_{t}\right) \doteq \log _{10}\left|1-e^{c^{(n)}\left(z_{t}\right)-c^{(0)}\left(z_{t}\right)}\right|
$$

where $c_{t}^{(n)}\left(z_{t}\right)$ solves equation $0=\ln E_{t} e^{m_{t+1}\left[c_{t+1}^{(n-1)}\left(z_{t}\right), c_{t}^{(n)}\left(z_{t}\right)\right]+r_{t}}$, for points $z_{t}$ that cover a high-probability region of the state space, and a stochastic discount factor $m_{t+1}$ that is a function of consumption. A $n$-period Euler equation error of $-\varepsilon$ implies that the consumer is making a one dollar mistake in how much she decides to save over a $n$-period horizon for every $10^{\varepsilon}$ dollars spent. Since errors accumulate as the horizon increase, multiperiod Euler equation errors provide an indication of how good the approximation is for long-term valuations.

\subsection{Endowment economy with habits (Campbell and Cochrane, 1999)}

Figure 4 complements figures 1 and 2 by comparing the global solution with our proposed affine solution. The figure reports the term structures of equilibrium risk premia and realized return volatilities of zero-coupon equities and bonds. Dividends are defined as in Campbell and Cochrane (1999). While figure 2 describes the approximation of how equilibrium prices depend on the state, figure 4 is based on simulations and hence it reflects both the approximation of the policy function and the approximation of the dynamics of state variables.

In this context, since the unique state is exogenous in this endowment economy, the standard loglinear-lognormal method and our affine approximation coincide (see footnotes 8 and 17). Relative to the projected solution, the fit of the generalized affine approximation manages to capture the level, amplitude, and shape of the term structures.

\subsection{Endowment economy with time-varying rare disasters (Wachter, 2013)}

Figure 5 complements figure 2 by comparing the global solution with our proposed affine solution. The figure reports the term structures of equilibrium risk premia and realized return volatilities of zero-coupon equities and bonds. Dividends are defined as in Wachter (2013) as levered consumption $D_{t}=C_{t}^{2.6}$.

Relative to the projected solution, the fit of the affine approximation is tight as it manages to capture the level, amplitude, and shape of the term structures. The presence of non-Gaussianities prevents the use of extant loglinear-lognormal methods. 


\begin{tabular}{lc}
\hline Parameter & Habit formation \\
\hline Frequency & quarterly \\
Subjective discount factor, $\beta$ & .987 \\
Utility curvature parameter $\gamma$ & 2 \\
Habit persistence, $\phi$ & .98 \\
Steady-state surplus consumption ratio, $S$ & .073 \\
Mean growth rate (in \%), $\mu$ & $1.80 / 4$ \\
Standard deviation of tfp innovations (in $\%), \sigma_{c}$ & $1.20 / \sqrt{4}$ \\
Capital share, $\alpha$ & 0.35 \\
Investment-capital ratio, $\bar{i}=\frac{\delta}{1+1 / \xi}$ & 0.0205 \\
Capital adjustment cost curvature, $\frac{1}{\xi}$ & 0.4 \\
\hline
\end{tabular}

Table 2: Deep parameters and their calibration (quarterly frequency) in the RBC model with Campbell-Cochrane habits.

\subsection{Production economy with habits (Jermann, 1998; Chen, 2017)}

A representative consumer with Campbell-Cochrane habits in consumption lives in a production economy and chooses output $Y_{t}=A_{t}^{1-\alpha} K_{t}^{\alpha}$ and the trajectory of capital, whose accumulation is subject to adjustment costs:

$$
K_{t+1}=\left[1-\delta+\Phi\left(\frac{I_{t}}{K_{t}}\right)\right] K_{t}=e^{\mu} K_{t}+\frac{\bar{i}}{1-\frac{1}{\xi}}\left[\left(\frac{I_{t}}{\bar{i} K_{t}}\right)^{1-\frac{1}{\xi}}-1\right] K_{t}
$$

where $\bar{i} \doteq \frac{\delta}{1+1 / \xi}$ is the deterministic steady-state investment-capital ratio. Output is devoted to consumption or to investment, $Y_{t}=C_{t}+I_{t}$. Technology and habits are driven by:

$$
\begin{aligned}
a_{t+1} & =\mu+a_{t}+\sigma \varepsilon_{t+1} \\
s_{t+1} & =(1-\phi) s+\phi s_{t}+\Lambda\left(\hat{s}_{t}\right)\left(c_{t+1}-E_{t} c_{t+1}\right)
\end{aligned}
$$

where $\varepsilon_{t} \sim \operatorname{Niid}(0,1)$ and $\Lambda\left(\hat{s}_{t}\right)=S^{-1} \sqrt{1-2 \hat{s}_{t}}-1$.

This specification is a version of the model in Jermann (1998) with Campbell-Cochrane habits explored recently by Chen (2017). Accordingly, we calibrate the model using the values listed in table 2 . We let the sensitivity function of surplus consumption vary to avoid a risk-free rate puzzle, and set $\beta$ and $S$ to achieve a stable risk-free rate around the mean reported by Chen. The rest of the parametrization is the same as in Chen (2017).

Figure 3 plots the policy function of equilibrium investment-capital ratio and consumptionproductivity ratio as function of the states. Deterministic and risky steady state values of states differ, especially for detrended capital $K / A$. Each plot sets the other state to its steady state value. In particular, the affine approximation is close to the global solution at the risky steady stateespecially as $S$ varies - whereas the deterministic steady state approximation is inaccurate. We also plot the standard loglinear-lognormal approximation in Malkhozov (2014), which disregards the volatility in the sensitivity function of surplus consumption due to the presence of endogenous 

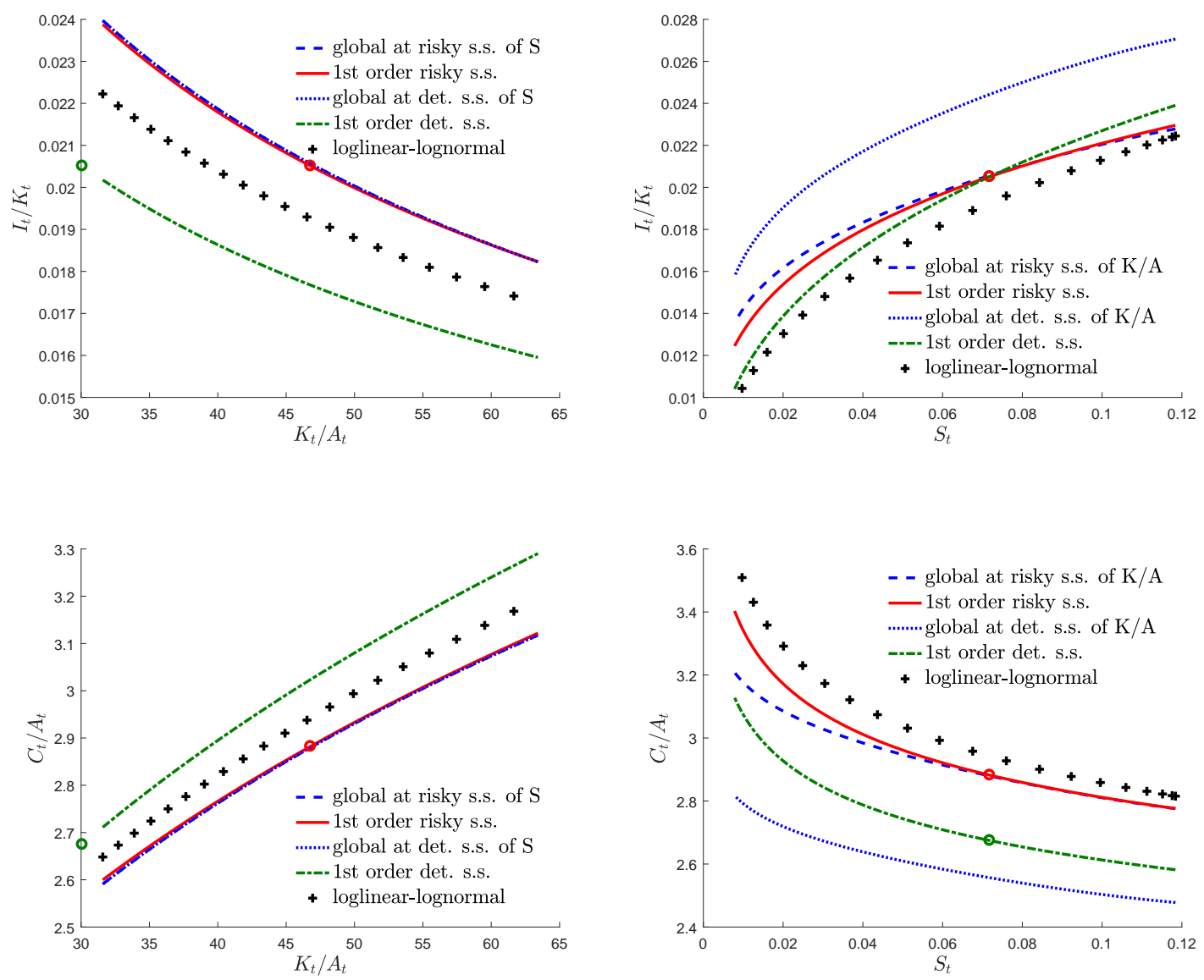

Figure 3: Comparison of first-order approximations around the risky and the deterministic steady state for the map of states into the investment-capital ratio and detrended consumption in the model with capital accumulation and Campbell-Cochrane habits. The projected solution uses Smolyak collocation of Chebyshev polynomials of up to degree 8 and 10-point Gauss-Hermite quadrature. The state space consists of surplus consumption $(S)$ and detrended capital $(K / A)$. Black and red circles denote deterministic and risky steady states. For a meaningful comparison given the local nature of perturbations, we plot the global solution as we vary one state and set the other at the risky and the deterministic steady state; similarly, risky and deterministic steady state approximations vary one state and set the other at the respective steady state. 


\section{(consumption) risk. ${ }^{17}$}

Figure 6 plots term structure implications; in this context, the risk-adjusted linearization performs similarly as the global solution. Risk pricing is accurate at all horizons.

Figure 7 shows multiperiod Euler equation errors. The accuracy of our global solution in terms of conventional 1-step ahead Euler equation errors is consistently lower than -2, and remains with maximums of around -2 over arbitrarily long horizons. These values are considerably lower than under the global solution but remain relatively small; values of around -3 are typically retained as acceptable in the extant literature (e.g., Fernández-Villaverde et al., 2015a).

\section{Conclusion}

Exponential-affine approximations have routinely been used in the finance literature as ad-hoc approximation strategies. As we show, the desirable implications of these methods find a formal justification in the theory of local perturbation approximations based on the implicit function and Taylor theorems. Our entropy-based approximation generalizes the applicability of the methodology while achieving a precise relation with perturbations around a stochastic stationary point. The resulting approximation technique offers explicit formulas and numerical routines to approximate equilibrium quantities and asset prices in a large class of dynamic macro-finance models as well as conditions for existence and uniqueness of the approximate local dynamics. We have also provided a flexible and user-friendly Matlab code available online.

\section{Appendix}

\section{A. Non-recursive utility}

We can express non-recursive utility in terms of forward-looking difference equations.

Non-recursive utility is defined as

$$
V_{t}=\left[(1-\beta) C_{t}^{1-\rho}+\beta\left(E_{t} V_{t+1}^{1-\gamma}\right)^{\frac{1-\rho}{1-\gamma}}\right]^{\frac{1}{1-\rho}}
$$

and hence $V_{t}\left(C_{t}, V_{t+1}\right)$ is homogeneous of degree one. It follows that by Euler's theorem we have

$$
V_{t}=\frac{\partial V_{t}}{\partial C_{t}} C_{t}+E_{t}\left(\frac{\partial V_{t}}{\partial V_{t+1}} V_{t+1}\right)
$$

with $\partial V_{t} / \partial C_{t}=(1-\beta) V_{t}^{\rho} C_{t}^{-\rho}$ and $\partial V_{t} / \partial V_{t+1}=\beta V_{t}^{\rho} X_{t}^{\gamma-\rho} V_{t+1}^{-\gamma}$, where $X_{t}^{1-\gamma}=E_{t} V_{t+1}^{1-\gamma}$. We have the stochastic discount factor $M_{t+1}=\frac{\partial V_{t+1} / \partial C_{t+1}}{\partial V_{t} / \partial C_{t}} \frac{\partial V_{t}}{\partial V_{t+1}}=e^{\ln (\beta)-\gamma \Delta c_{t+1}-(\gamma-\rho)\left(v c_{t+1}-x c_{t}\right)}$.

\footnotetext{
${ }^{17}$ To treat the standard loglinear-lognormal fairly, we apply it to the specification of the model with large $N$, even though the standard method never realized the existence of a different approximation of the problem for each $N$. For $N=1$, the default specification of the standard method, the performance of the loglinear-lognormal specification would worsen substantially, and become much closer to the deterministic steady-state approximation.
} 
Next, define variable $W_{t} \equiv \frac{V_{t}}{\partial V_{t} / \partial C_{t}}$, and note that, using Euler's theorem,

$$
\begin{aligned}
W_{t}=\frac{V_{t}}{\partial V_{t} / \partial C_{t}} & =C_{t}+E_{t} \frac{\partial V_{t+1} / \partial C_{t+1}}{\partial V_{t} / \partial C_{t}} \frac{\partial V_{t}}{\partial V_{t+1}} \frac{V_{t+1}}{\partial V_{t+1} / \partial C_{t+1}}=C_{t}+E_{t} M_{t+1} W_{t+1} \\
& =E_{t} M_{t, t+N} W_{t+N}+\sum_{n=0}^{N-1} E_{t} M_{t, t+n} C_{t+n}
\end{aligned}
$$

Therefore, $W_{t}$ is wealth-the price of a claim to the stream of future consumption.

Thus, a complete description of recursive utility is:

$$
\begin{aligned}
v_{t}-c_{t} & =\frac{\ln (1-\beta)}{1-\rho}+\frac{1}{1-\rho}\left(w_{t}-c_{t}\right) \\
x_{t}-c_{t} & =\frac{1}{1-\rho} \ln \left(e^{w_{t}-c_{t}}-1\right)+\frac{\ln (1-\beta)-\ln (\beta)}{1-\rho} \\
w_{t}-c_{t} & =\ln \left(e^{r c_{t}^{(N)}}+\sum_{n=0}^{N-1} e^{p c_{t}^{(n)}}\right), \quad e^{r c_{t}^{(n)}}=E_{t} e^{m_{t+1}+\Delta c_{t+1}+r c_{t+1}^{(n-1)}}, \quad e^{p c_{t}^{(n)}}=E_{t} e^{m_{t+1}+\Delta c_{t+1}+p c_{t+1}^{(n-1)}}
\end{aligned}
$$

with boundary conditions $p c_{t}^{(0)}=0$ and $r c_{t}^{(0)}=w c_{t}$, where the log wealth-consumption ratio solves a forward-looking difference equation.

\section{B. Proof of proposition 1.a}

We follow Klein (2000) and consider the generalized Schur factorization of $\Gamma$ and $\Xi^{q}$, with unitary $Q, Z \in \mathbb{C}^{n_{y}+n_{z} \times n_{y}+n_{z}}$ and upper triangular matrices $S, T \in \mathbb{C}^{n_{y}+n_{z} \times n_{y}+n_{z}}$ such that:

$$
Q \Gamma Z=S=\left[\begin{array}{cc}
S_{11} & S_{12} \\
0 & S_{22}
\end{array}\right] \quad Q \Xi^{q} Z=T=\left[\begin{array}{cc}
T_{11} & T_{12} \\
0 & T_{22}
\end{array}\right] \quad Z=\left[\begin{array}{ll}
Z_{11} & Z_{12} \\
Z_{21} & Z_{22}
\end{array}\right], Z^{*}=\left[\begin{array}{cc}
Z_{11}^{*} & Z_{21}^{*} \\
Z_{12}^{*} & Z_{22}^{*}
\end{array}\right]
$$

with $Z^{*}$ the conjugate transpose of $Z$, where $S_{11}, T_{11} \in \mathbb{C}^{n_{z} \times n_{z}}, S_{22}, T_{22} \in \mathbb{C}^{n_{y} \times n_{y}}, Z_{11} \in \mathbb{C}^{n_{z} \times n_{z}}, Z_{12} \in$ $\mathbb{C}^{n_{z} \times n_{y}}$, and matrices $S, T$ are sorted with generalized eigenvalues $\alpha(\Gamma, \Xi)=\left\{t_{i i} / s_{i i}, i=1, \ldots, n_{y}+n_{z}\right\}$ in increasing order as $\left|t_{i i} / s_{i i}\right|<1, i=1, \ldots, n_{z}$ and $\left|t_{i i} / s_{i i}\right|>1, i=n_{z}+1, \ldots, n_{z}+n_{y}$. The dependence of $Q, S, T, Z$ on $q$ is not denoted explicitly for simplicity.

We rewrite the matrix equation that describes the affine solution (17) as:

$$
\begin{gathered}
\Gamma\left[\begin{array}{l}
I_{n_{z}} \\
\Psi^{q}
\end{array}\right]\left[g_{1}\left(y^{q}, z^{q}\right) \Psi^{q}+g_{2}\left(y^{q}, z^{q}\right)\right]\left(z_{t}^{q}-z^{q}\right)=\Xi\left[\begin{array}{l}
I_{n_{z}} \\
\Psi^{q}
\end{array}\right]\left(z_{t}^{q}-z^{q}\right) \\
\text { or: } Q \Gamma Z Z^{*}\left[\begin{array}{l}
I_{n_{z}} \\
\Psi^{q}
\end{array}\right] E_{t}\left(z_{t+1}^{q}-z^{q}\right)=Q \Xi Z Z^{*}\left[\begin{array}{l}
I_{n_{z}} \\
\Psi^{q}
\end{array}\right]\left(z_{t}^{q}-z^{q}\right) \Leftrightarrow \quad
\end{gathered}
$$

with

$$
\left[\begin{array}{c}
x_{z, t} \\
x_{y, t}
\end{array}\right] \doteq Z^{*}\left[\begin{array}{l}
I_{n_{z}} \\
\Psi^{q}
\end{array}\right]\left(z_{t}^{q}-z^{q}\right), \quad x_{z, t} \in \mathbb{R}_{t}^{n_{z}}, x_{y, t} \in \mathbb{R}_{t}^{n_{y}}
$$


Note that the upper triangular matrices $S_{11}$ and $T_{22}$ are invertible, as their respective eigenvalues $\left\{s_{i i}, i=1, \ldots, n_{z}\right\}$ and $\left\{t_{i i}, i=n_{z}+1, \ldots, n_{z}+n_{y}\right\}$ are nonzero by the assumption about eigenvalues.

By the stability requirement $\lim \left|E_{t} z_{t+N}^{q}\right|<\infty$, equation (B.1) implies:

$$
x_{y, t}=T_{22}^{-1} S_{22} E_{t} x_{y, t+1}=\left(T_{22}^{-1} S_{22}\right)^{N} E_{t} x_{y, t+N} \stackrel{N \rightarrow \infty}{\rightarrow} 0
$$

as the eigenvalues of the upper triangular matrix $T_{22}^{-1} S_{22}$ coincide with $\left\{s_{i i} / t_{i i}, i=n_{z}+1, \ldots, n_{z}+n_{y}\right\}$, hence lie within the unit circle. Therefore, $x_{y, t}$ is determined uniquely and is a bounded process if and only if $\left\{s_{i i} / t_{i i}, i=n_{z}+1, \ldots, n_{z}+n_{y}\right\}$ lie within the unit circle.

Next, using definition (B.2), it follows that $\Psi^{q}=-\left(Z_{22}^{*}\right)^{-1} Z_{12}^{*}=Z_{21} Z_{11}^{-1}$, where the last equality and invertibility owe to the orthonormality of matrix $Z$. Orthonormality of $Z$ also implies $Z_{11}^{*}-$ $Z_{21}^{*}\left(Z_{22}^{*}\right)^{-1} Z_{12}^{*}=Z_{11}^{-1}$. Therefore, equation (B.1) implies:

$$
E_{t} x_{z, t+1}=S_{11}^{-1} T_{11} x_{z, t}, \quad x_{z, t}=\left(Z_{11}^{*}+Z_{21}^{*} \Psi^{q}\right)\left(z_{t}^{q}-z^{q}\right)=Z_{11}^{-1}\left(z_{t}^{q}-z^{q}\right)
$$

hence $E_{t}\left(z_{t+1}^{q}-z^{q}\right)=Z_{11} S_{11}^{-1} T_{11} Z_{11}^{-1}\left(z_{t}^{q}-z^{q}\right)$, so the spectrum of matrix $g_{1}\left(y^{q}, 0\right) \Psi^{q}+g_{2}\left(y^{q}, 0\right)$ is:

$$
\left\{\lambda \in \mathbb{C}: \operatorname{det}\left[Z_{11} S_{11}^{-1} T_{11} Z_{11}^{-1}-\lambda I_{n_{z}}\right]=0\right\}=\left\{\frac{t_{i i}}{s_{i i}}, i=1, \ldots, n_{z}\right\}
$$

Therefore, the state vector has stable dynamics if and only if $\left\{t_{i i} / s_{i i}, i=1, \ldots, n_{z}\right\}$ lie within the unit circle.

\section{Proof of proposition 1.b}

We are looking for functionals $y_{t}^{q}=y\left(z_{t}^{q}, q, \tau\right)$ and $z_{t+1}^{q}=z\left(z_{t}^{q}, q, \varepsilon_{t+1}, \tau\right)$, and we rewrite the dynamic system in functional form as:

$$
F\left(\left[y^{q}, z^{q}\right], \varepsilon, q, \tau\right)=\left\{\left[\begin{array}{c}
h\left(y_{t}^{q}, z_{t}^{q}\right)+f_{3} E_{t} y_{t+1}^{q}+f_{4} E_{t} z_{t+1}^{q}+\tau w\left(z_{t}^{q}, q, \tau\right)+(1-\tau) \widetilde{\mathcal{V}}\left(z_{t}^{q}, q\right) \\
z_{t+1}^{q}-g\left(y_{t}^{q}, z_{t}^{q}\right)-\lambda\left(z_{t}^{q}\right)\left(y_{t+1}^{q}-E_{t} y_{t+1}^{q}\right)-q \sigma\left(z_{t}^{q}\right) \varepsilon_{t+1}
\end{array}\right]\right\}_{t=0}^{\infty}
$$

whose $t$ th coordinate maps $q \in[0,1], \tau \in[0,1], \varepsilon_{t+1} \in \mathbb{R}^{n_{\varepsilon}}$ and essentially-bounded functions $\left[y_{t}^{q} ; z_{t}^{q} ; y_{t+1}^{q} ; z_{t+1}^{q}\right]$ of the history of shocks $\left\{\varepsilon_{s}\right\}_{s \leq t+1}$ into the Banach space of essentially-bounded functions of the history of shocks $\left\{\varepsilon_{s}\right\}_{s \leq t}$. The derivative operator of map $F$ evaluated at the expansion point has $t$ th coordinate:

$$
D_{F, t}\left[\hat{y}^{q} ; \hat{z}^{q}\right]=\Gamma\left[\begin{array}{c}
E_{t} \hat{z}_{t+1}^{q} \\
E_{t} \hat{y}_{t+1}^{q}
\end{array}\right]-\Xi^{q}\left[\begin{array}{c}
\hat{z}_{t}^{q} \\
\hat{y}_{t}^{q}
\end{array}\right],
$$

and it maps an a.s.-bounded sequence of perturbed arguments $\left\{\hat{y}_{t}^{q} ; \hat{z}_{t}^{q}\right\}_{t=0}^{\infty}$ into a unique a.s.-bounded process $u^{q}=\left\{u_{t}^{q}\right\}_{t=0}^{\infty}$ that is a measurable function of the history of shocks. Note how the derivative operator is well-defined because the ccgf of exogenous shocks exists and is differentiable. ${ }^{18}$

\footnotetext{
${ }^{18}$ Also, the existence of the ccgf of exogenous shocks is not a local property and yet is a necessary regularity condition, as the moment $E_{t} e^{\alpha\left(z_{t}\right) q \varepsilon_{t+1}}$ for a real map $\alpha$ needs not exist otherwise even for arbitrarily small $q>0$. Jin and Judd (2002) and Kim et al. (2008) make a similar point about the existence of moments of shocks.
} 
Risky steady state is a saddle point $\Rightarrow$ Locally unique and differentiable implicit functions. The goal is to show that maps $\left[y^{q}, z^{q}\right]$ of $[\varepsilon, q, \tau]$ are defined uniquely and are differentiable on a sufficiently small neighborhood of $[0, q, 0]$. If we can invoke the implicit function theorem, the proof follows immediately. To be able to invoke the implicit function theorem in Banach spaces (e.g. Lang, 1993, 364), we have to prove that the derivative operator around the expansion point is invertible as a continuous (and hence bounded) linear operator. ${ }^{19}$

In turn, we have invertibility-i.e., an a.s.-bounded process $\left\{u_{t}^{q}\right\}_{t=0}^{\infty}$ maps into unique a.s.bounded processes $\left\{\hat{y}_{t}^{q} ; \hat{z}_{t}^{q}\right\}_{t=0}^{\infty}$-if and only if the expansion point is a saddle point. To prove this claim, we write the derivative as:

$$
Q D_{F, t}\left[\hat{y}^{q} ; \hat{z}^{q}\right]=S\left[\begin{array}{l}
E_{t} x_{z, t+1} \\
E_{t} x_{y, t+1}
\end{array}\right]-T\left[\begin{array}{l}
x_{z, t} \\
x_{y, t}
\end{array}\right] \text {, with }\left[\begin{array}{l}
x_{z, t} \\
x_{y, t}
\end{array}\right] \doteq Z^{*}\left[\begin{array}{c}
\hat{z}_{t}^{q} \\
\hat{y}_{t}^{q}
\end{array}\right]
$$

where $Q, S, T, Z$ constitute the Schur factorization of $\Gamma$ and $\Xi^{q}$. The dependence of $Q, S, T, Z$ on $q$ is not denoted explicitly for simplicity. We then note that the derivative operator in equation:

$$
D_{F}\left[\hat{y}^{q} ; \hat{z}^{q}\right]=u^{q} \quad \Leftrightarrow \quad S\left[\begin{array}{l}
E_{t} x_{z, t+1} \\
E_{t} x_{y, t+1}
\end{array}\right]=T\left[\begin{array}{l}
x_{z, t} \\
x_{y, t}
\end{array}\right]+v_{t}^{q}, \quad\left[\begin{array}{c}
v_{z, t}^{q} \\
v_{y, t}^{q}
\end{array}\right] \doteq Q u_{t}^{q}
$$

can be inverted as:

$$
\begin{aligned}
x_{y, t} & =T_{22}^{-1} S_{22} E_{t} x_{y, t+1}-T_{22}^{-1} v_{y, t}^{q} \stackrel{N \rightarrow \infty}{\rightarrow}-\sum_{j=0}^{\infty}\left(T_{22}^{-1} S_{22}\right)^{j} T_{22}^{-1} E_{t} v_{y, t+j}^{q} \\
E_{t} x_{z, t+1} & =S_{11}^{-1} T_{11} x_{z, t}+S_{11}^{-1}\left(T_{12} x_{y, t}-S_{12} E_{t} x_{y, t+1}\right)+S_{11}^{-1} v_{z, t}^{q}
\end{aligned}
$$

if and only if $T_{22}$ and $S_{11}$ are invertible and $T_{22}^{-1} S_{22}$ and $S_{11}^{-1} T_{11}$ have eigenvalues inside the unit circle; this property defines the FRSS as a saddle point. Orthonormal matrices $Q$ and $Z$ map $v^{q}$ and $\left[x_{z} ; x_{y}\right]$ back into the original processes $u^{q}$ and $\left[y^{q} ; z^{q}\right]$.

The invertibility of the derivative operator evaluated at the expansion point implies that we can rely on the implicit function theorem to characterize the functions of the history of shocks with the target form $y_{t}^{q}=y\left(z_{t}^{q}, q, \tau\right)$ and $z_{t+1}^{q}=z\left(z_{t}^{q}, q, \varepsilon_{t+1}, \tau\right)$ that solve $F\left(\left[y^{q}, z^{q}\right], \varepsilon, q, \tau\right)=0$. Namely, these functions are unique and differentiable in a neighborhood of the expansion point $\left(z_{t}, \tau\right)=\left(z^{q}, 0\right)$.

Locally unique and differentiable implicit functions $\Rightarrow$ Coefficients from first-order Taylor approximation equal coefficients from affine approximation. The uniqueness and differentiability of the implicit functions implies that we can now approximate the local solution around the FRSS $\left(z_{t}^{q}, \tau\right)=\left(z^{q}, 0\right)$ via Taylor theorem. (Note how no expansion in $q$ will take place.) We are looking

\footnotetext{
${ }^{19}$ We also require $\left[y^{q}, z^{q}\right]$ to be in an open set of the topology of a.s.-bounded functions. As in the case of linear perturbations around the deterministic steady state, we can guarantee this property in the topology of essentiallybounded functions if exogenous shocks have a.s.-bounded support (Jin and Judd, 2002). Note that the reasoning is local; in particular, for a $z_{t}^{q}$ in a neighborhood of $\widetilde{z}^{q}$ we have that $z_{t+1}^{q}$ is in the same neighborhood only under a sufficiently small $q>0$. Whether $q=1$ is sufficiently small will in turn depend on whether $\sigma\left(z^{q}\right)$ is and will be a practical question about the quality of the approximation.
} 
to identify the approximate functions:

$$
\begin{aligned}
y_{t}^{q} & =y\left(\widetilde{z}^{q}, q, 0\right)+y_{1}\left(\bar{z}^{q}, q, 0\right)\left(z_{t}^{q}-\widetilde{z}^{q}\right)+y_{3}\left(\widetilde{z}^{q}, q, 0\right) \tau \\
z_{t+1}^{q} & =z\left(\widetilde{z}^{q}, q, \varepsilon_{t+1}, 0\right)+z_{1}\left(\widetilde{z}^{q}, q, \varepsilon_{t+1}, 0\right)\left(z_{t}^{q}-\widetilde{z}^{q}\right)+z_{4}\left(\widetilde{z}^{q}, q, \varepsilon_{t+1}, 0\right) \tau \\
x_{t+1}^{q} & =x\left(\bar{z}^{q}, q, \varepsilon_{t+1}, 0\right)+x_{1}\left(\widetilde{z}^{q}, q, \varepsilon_{t+1}, 0\right)\left(z_{t}^{q}-\widetilde{z}^{q}\right)+x_{4}\left(\bar{z}^{q}, q, \varepsilon_{t+1}, 0\right) \tau
\end{aligned}
$$

It is useful to define the derivative of a differentiable matrix $\lambda: \mathbb{R}^{n_{z}} \rightarrow \mathbb{R}^{n_{y} \times n_{x}}$ as:

$$
\lambda_{1}(0)=\left[\begin{array}{c}
\frac{\partial \lambda_{(1, i)}(0)}{\partial \hat{z}_{t}} \\
\vdots \\
\frac{\partial \lambda_{\left(n_{y, j}\right)}(0)}{\partial \hat{z}_{t}}
\end{array}\right] \in \mathbb{R}^{n_{z} n_{y} \times n_{x}}, \quad \frac{\partial \lambda_{(i,:)}(0)}{\partial \hat{z}_{t}} \doteq\left[\begin{array}{ccc}
\frac{\partial \lambda_{(i, 1)}(0)}{\partial \hat{z}_{1, t}} & \cdots & \frac{\partial \lambda_{\left(i, n_{x}\right)}(0)}{\partial \hat{z}_{1, t}} \\
\vdots & & \vdots \\
\frac{\partial \lambda_{(i, 1)}(0)}{\partial \hat{z}_{n, t}} & \cdots & \frac{\partial \lambda_{\left(i, n_{x}\right)}(0)}{\partial \hat{z}_{n_{z}, t}}
\end{array}\right] \in \mathbb{R}^{n_{z} \times n_{x}}
$$

for each row $i=1, \ldots, n_{z}$.

A Taylor approximation of the equilibrium conditions around point $\left[z_{t}^{q}, \tau\right]=\left[\bar{z}^{q}, 0\right]$ yields:

$$
\begin{aligned}
& z\left(z^{q}, q, \varepsilon_{t+1}, 0\right)=g\left[y\left(z^{q}, q, 0\right), \bar{z}^{q}\right]+\lambda\left(z^{q}\right)\left(E_{t+1}-E_{t}\right) y\left[z\left(z^{q}, q, \varepsilon_{t+1}, 0\right), q, 0\right]+q \sigma\left(z^{q}\right) \varepsilon_{t+1} \\
& =g\left[y\left(z^{q}, q, 0\right), \bar{z}^{q}\right]+\sigma_{z}\left(z^{q}, q\right) \varepsilon_{t+1}, \quad \sigma_{z}\left(z_{t}^{q}, q\right) \doteq q\left[I_{n_{z}}-\lambda\left(z_{t}^{q}\right) y_{1}\left(z^{q}, q, 0\right)\right]^{-1} \sigma\left(z_{t}^{q}\right) \\
& z_{1}\left(z^{q}, q, \varepsilon_{t+1}, 0\right)\left(z_{t}^{q}-\bar{z}^{q}\right)=\left[g_{1}^{q} y_{1}\left(z^{q}, q, 0\right)+g_{2}^{q}\right]\left(z_{t}^{q}-\bar{z}^{q}\right)+\left[I_{n_{z}} \otimes\left(z_{t}^{q}-\bar{z}^{q}\right)^{\prime}\right] \lambda_{1}\left(z^{q}\right)\left(E_{t+1}-E_{t}\right) y\left[z\left(z^{q}, q, \varepsilon_{t+1}, 0\right), q, 0\right] \\
& +\lambda\left(z^{q}\right)\left(E_{t+1}-E_{t}\right) y_{1}\left[z\left(z^{q}, q, \varepsilon_{t+1}, 0\right), q, 0\right] z_{1}\left(z^{q}, q, \varepsilon_{t+1}, 0\right)\left(z_{t}^{q}-\bar{z}^{q}\right) \\
& +\left[I_{n_{z}} \otimes\left(z_{t}^{q}-\bar{z}^{q}\right)^{\prime}\right] \sigma_{1}\left(z^{q}\right) q \varepsilon_{t+1} \\
& =\left[g_{1}^{q} y_{1}\left(z^{q}, q, 0\right)+g_{2}^{q}\right]\left(z_{t}^{q}-\bar{z}^{q}\right)+\left[I_{n_{z}} \otimes\left(z_{t}^{q}-\bar{z}^{q}\right)^{\prime}\right] \sigma_{1, z}\left(\bar{z}^{q}, q\right) \varepsilon_{t+1} \\
& z_{4}\left(z^{q}, q, \varepsilon_{t+1}, 0\right)=g_{1}^{q} y_{3}\left(z^{q}, q, 0\right)+\left[I_{n_{z}}-\lambda\left(z_{t}^{q}\right) y_{1}\left(z^{q}, q, 0\right)\right]^{-1} \lambda\left(z^{q}\right)\left(E_{t+1}-E_{t}\right) y_{3}\left(z^{q}, q, 0\right) \\
& =g_{1}^{q} y_{3}\left(z^{q}, q, 0\right)
\end{aligned}
$$

where $g_{1}^{q} \doteq g_{1}\left[y\left(\widetilde{z}^{q}, q, 0\right), \widetilde{z}^{q}\right]$ and $g_{2}^{q} \doteq g_{2}\left[y\left(\widetilde{z}^{q}, q, 0\right), \widetilde{z}^{q}\right]$, with the auxiliary variable:

$$
\begin{aligned}
x\left(z^{q}, q, \varepsilon_{t+1}, 0\right)= & h\left[y\left(z^{q}, q, 0\right), \bar{z}^{q}\right]+f_{3} y\left[z\left(\bar{z}^{q}, q, \varepsilon_{t+1}, 0\right), q, 0\right]+f_{4} z\left(\bar{z}^{q}, q, \varepsilon_{t+1}, 0\right) \\
= & h\left[y\left(\bar{z}^{q}, q, 0\right), \bar{z}^{q}\right]+f_{3} y\left(z^{q}, q, 0\right)+f_{4} \bar{z}^{q}+\left[f_{3} y_{1}\left(\bar{z}^{q}, q, 0\right)+f_{4}\right] \sigma_{z}\left(\bar{z}^{q}, q\right) \varepsilon_{t+1} \\
x_{1}\left(z^{q}, q, \varepsilon_{t+1}, 0\right)\left(z_{t}^{q}-\widetilde{z}^{q}\right)= & {\left[f_{1}^{q} y_{1}\left(z^{q}, q, 0\right)+f_{2}^{q}+f_{3} y_{1}\left[z\left(\bar{z}^{q}, q, \varepsilon_{t+1}, 0\right), q, 0\right] z_{1}\left(z^{q}, q, \varepsilon_{t+1}, 0\right)+f_{4} z_{1}\left(\bar{z}^{q}, q, \varepsilon_{t+1}, 0\right)\right]\left(z_{t}^{q}-\widetilde{z}^{q}\right) } \\
= & {\left[f_{1}^{q} y_{1}\left(z^{q}, q, 0\right)+f_{2}^{q}+\left[f_{3} y_{1}\left(z^{q}, q, 0\right)+f_{4}\right]\left(g_{1}^{q} y_{1}\left(z^{q}, q, 0\right)+g_{2}^{q}\right)\right]\left(z_{t}^{q}-\widetilde{z}^{q}\right) } \\
& \quad+\left[f_{3} y_{1}\left(z^{q}, q, 0\right)+f_{4}\right]\left[I_{n_{z}} \otimes\left(z_{t}^{q}-\widetilde{z}^{q}\right)^{\prime}\right] \sigma_{1, z}\left(z^{q}, q\right) \varepsilon_{t+1} \\
x_{4}\left(z^{q}, q, \varepsilon_{t+1}, 0\right)= & f_{1}^{q} y_{3}\left(z^{q}, q, 0\right)+f_{3} y_{3}\left[z\left(z^{q}, q, \varepsilon_{t+1}, 0\right), q, 0\right]+\left[f_{3} y_{1}\left[z\left(z^{q}, q, \varepsilon_{t+1}, 0\right), q, 0\right]+f_{4}\right] z_{4}\left(z^{q}, q, \varepsilon_{t+1}, 0\right) \\
= & {\left[f_{1}^{q}+f_{3}+\left[f_{3} y_{1}\left(z^{q}, q, 0\right)+f_{4}\right] g_{1}^{q}\right] y_{3}\left(\bar{z}^{q}, q, 0\right) }
\end{aligned}
$$

where $f_{1}^{q} \doteq f_{1}\left[y\left(\widetilde{z}^{q}, q, 0\right), \widetilde{z}^{q}\right]$ and $f_{2}^{q} \doteq f_{2}\left[y\left(\widetilde{z}^{q}, q, 0\right), \widetilde{z}^{q}\right]$. In the derivation we used the property of the approximate solution:

$$
\begin{aligned}
y\left[z\left(\bar{z}^{q}, q, \varepsilon_{t+1}, 0\right), q, 0\right] & =y\left(\bar{z}^{q}, q, 0\right)+y_{1}\left(\widetilde{z}^{q}, q, 0\right) \sigma_{z}\left(z^{q}, q\right) \varepsilon_{t+1} \\
y_{1}\left[z\left(z^{q}, q, \varepsilon_{t+1}, 0\right), q, 0\right] & =y_{1}\left(z^{q}, q, 0\right)
\end{aligned}
$$

that follows from $y\left[z\left(z_{t}^{q}, q, \varepsilon_{t+1}, 0\right), q, 0\right]=y\left(\widetilde{z}^{q}, q, 0\right)+y_{1}\left(\widetilde{z}^{q}, q, 0\right)\left[z\left(z_{t}^{q}, q, \varepsilon_{t+1}, 0\right)-\widetilde{z}^{q}\right]$. 
Next, we evaluate entropy using the local solution:

$$
w\left(\bar{z}^{q}, q, 0\right)=\mathcal{V}\left[e^{x\left(\bar{z}^{q}, q, \varepsilon_{t+1}\right)+x_{1}\left(\bar{z}^{q}, q, \varepsilon_{t+1}\right)\left(z_{t}^{q}-\bar{z}^{q}\right)} \widetilde{z}^{q}\right]=\kappa\left[\left(f_{3} y_{1}\left(\bar{z}^{q}, q, 0\right)+f_{4}\right) \sigma_{z}\left(\bar{z}^{q}, q\right) ; \widetilde{z}^{q}\right]
$$

and hence we identify $\left[y\left(\widetilde{z}^{q}, q, 0\right), y_{1}\left(\widetilde{z}^{q}, q, 0\right)\right]$ using equation $E_{t} x_{t+1}^{q}+\tau w\left(z_{t}^{q}, q, \tau\right)+(1-\tau) \widetilde{\mathcal{V}}\left(z_{t}^{q}, q\right)=0$ and matching coefficients as:

$$
\begin{aligned}
& 0=h\left[y\left(z^{q}, q, 0\right), \widetilde{z}^{q}\right]+f_{3} y\left(z^{q}, q, 0\right)+f_{4} \widetilde{z}^{q}+\widetilde{\mathcal{V}}\left(z^{q}, q\right) \\
& 0=f_{1}^{q} y_{1}\left(\widetilde{z}^{q}, q, 0\right)+f_{2}^{q}+\left[f_{3} y_{1}\left(\widetilde{z}^{q}, q, 0\right)+f_{4}\right]\left[g_{1}^{q} y_{1}\left(\widetilde{z}^{q}, q, 0\right)+g_{2}^{q}\right]+\widetilde{\mathcal{V}}_{1}\left(z^{q}, q\right) \\
& 0=\left[f_{1}^{q}+f_{3}+\left[f_{3} y_{1}\left(z^{q}, q, 0\right)+f_{4}\right] g_{1}^{q}\right] y_{3}\left(\widetilde{z}^{q}, q, 0\right)+w\left(z^{q}, q, 0\right)-\widetilde{\mathcal{V}}\left(\widetilde{z}^{q}, q\right)
\end{aligned}
$$

Matrix equations (C.4) and (C.5) coincide with matrix equation (17). It follows that $z^{q}=\widetilde{z}^{q}$, $y^{q}=y\left(z^{q}, 1,0\right)$ and $\Psi^{q}=y_{1}\left(z^{q}, 1,0\right)$. Therefore, matrix equations (C.4) and (C.5) at $q=1$ coincide with matrix equation (5); affine coefficients can be interpreted as the coefficients from a first-order perturbation around the $\operatorname{FRSS}\left(z_{t}^{q}, \tau\right)=\left(\widetilde{z}^{q}, 0\right)$ evaluated at $q \in[0,1]$ and $\varepsilon_{t+1}=0$.

Finally, $z^{q}=\widetilde{z}^{q}$ and $\Psi^{q}=y_{1}\left(\widetilde{z}^{q}, 1,0\right)$ imply $w\left(z^{q}, q, 0\right)=\widetilde{\mathcal{V}}\left(\widetilde{z}^{q}, q\right)$ by equation (C.3). It follows that $y_{3}\left(z^{q}, q, 0\right)=0$ by equation (C.6). The local slope of the solution with respect to $\tau$ is zero.

\section{Proof of proposition 3}

We conjecture that the price-dividend ratio of the $n$-period ahead cashflow strip has the exponential-affine shape $P_{d, t}^{(n)} / D_{t}=e^{A^{(n)}+B_{z}^{(n)} \hat{z}_{t}}$, and use the no-arbitrage relation:

$$
P_{d, t}^{(n)}=E_{t}^{\mathbb{P}}\left[e^{m_{t+1}} P_{d t+1}^{(n-1)}\right], \quad P_{d, t}^{(0)}=D_{t}
$$

to verify the conjecture as:

$$
\begin{aligned}
e^{A^{(n)}+B_{z}^{(n)} \hat{z}_{t}}=E_{t}^{\mathbb{P}}\left[e^{\left.m_{t+1}+A^{(n-1)}+B_{z}^{(n-1)} \hat{z}_{t+1}+\Delta d_{t+1}\right]}\right. \\
=e^{-r\left(\hat{z}_{t}\right)+\kappa\left[-\gamma\left(\hat{z}_{t}\right)^{\prime} ; z_{t}\right]+A^{(n-1)}+B_{z}^{(n-1)} A \hat{z}_{t}+\mu_{d}+C \hat{z}_{t}+\mathcal{V}_{t}\left[\exp \left(-\gamma\left(\hat{z}_{t}\right)^{\prime} \varepsilon_{t+1}+B_{z}^{(n-1)} B\left(\hat{z}_{t}\right) \varepsilon_{t+1}+D\left(\hat{z}_{t}\right) \varepsilon_{t+1}\right)\right]} \\
=e^{-r(0)-r_{1}(0) \hat{z}_{t}+A^{(n-1)}+B_{z}^{(n-1)} A \hat{z}_{t}+\mu_{d}+C \hat{z}_{t}+\kappa\left[-\gamma(0)^{\prime} ; z\right]+\left(\kappa_{1}\left[-\gamma(0)^{\prime} ; z\right] \gamma_{1}(0)+\kappa_{2}[-\gamma(0) ; z]\right) \hat{z}_{t}} \times \\
\quad \times e^{\kappa\left[-\gamma(0)^{\prime}+V_{n-1}(0)^{\prime} ; z\right]+\left(\kappa_{1}\left[-\gamma(0)^{\prime}+V_{n-1}(0)^{\prime} ; z_{t}\right]\left[-\gamma_{1}(0)+V_{1, n-1}(0)\right]+\kappa_{2}\left[-\gamma(0)^{\prime}+V_{n-1}(0)^{\prime} ; z\right]\right) \hat{z}_{t}}
\end{aligned}
$$

Matching coefficients, the initial guess can be identified as the solution of matrix equation (20). It is straightforward to recombine the affine approximations of the return components to derive:

$$
\ln E_{t}^{\mathbb{P}}\left[R_{d, t+1}^{(n)}\right] \equiv \ln E_{t}^{\mathbb{P}}\left[e^{p_{d, t+1}^{(n-1)}-p_{d, t}^{(n)}}\right]=r_{t}+C_{t}\left(M_{t+1}, R_{d, t+1}^{(n)}\right)
$$

Hold-to-maturity risk premia can be derived from the equilibrium expression for yields and the term structure of cashflow growth, $G_{d, t}^{(n)} \equiv E_{t}^{\mathbb{P}}\left[D_{t+n} / D_{t}\right]$, which has the recursive structure

$$
G_{d, t}^{(n)}=E_{t}^{\mathbb{P}}\left(\frac{D_{t+1}}{D_{t}} G_{d, t+1}^{(n-1)}\right)
$$


with boundary condition $G_{d, t}^{(0)}=1$, and hence implies $G_{d, t}^{(n)}=e^{A_{g}^{(n)}+B_{g}^{(n)} \hat{z}_{t}}$ up to a second-order term.

\section{E. Proof of proposition 4}

Assumption (18) implies the approximate joint ccgf:

$$
\ln E_{t}^{\mathbb{P}}\left[e^{u_{z}^{\prime} \hat{z}_{t+1}+u_{d} \Delta d_{t+1}}\right]=u^{\prime} \mu+\kappa\left[u^{\prime} \Sigma\left(\hat{z}_{t}\right) ; z_{t}\right]+u^{\prime} \Phi \hat{z}_{t},
$$

for $u=\left[u_{z} ; u_{d}\right] \in \mathbb{R}^{n_{z}+1}$. We define the multiplicative martingale,

$$
Q_{t+1}=Q_{t} e^{-\kappa\left[-\gamma\left(\hat{z}_{t}\right) ; z_{t}\right]-\gamma\left(\hat{z}_{t}\right)^{\prime} \varepsilon_{t+1}},
$$

to construct the change of measure from physical to risk-neutral probabilities, $d \mathbb{Q} / d \mathbb{P}$.

It follows that the risk-neutral dynamics of the vector process $[z ; \Delta d]$ are:

$$
\begin{aligned}
\ln E_{t}^{\mathbb{Q}}\left[e^{\left.u_{z}^{\prime} \hat{z}_{t+1}+u_{d} \Delta d_{t+1}\right]}\right. & =\ln E_{t}^{\mathbb{P}}\left[e^{-\kappa\left[-\gamma\left(\hat{z}_{t}\right)^{\prime} ; z_{t}\right]-\gamma\left(\hat{z}_{t}\right)^{\prime} \varepsilon_{t+1}+u_{z}^{\prime} \hat{z}_{t+1}+u_{d} \Delta d_{t+1}}\right] \\
& =-\kappa\left[-\gamma\left(\hat{z}_{t}\right)^{\prime} ; z_{t}\right]+u_{z}^{\prime} E_{t}^{\mathbb{P}} \hat{z}_{t+1}+u_{d} E_{t}^{\mathbb{P}} \Delta d_{t+1}+\mathcal{V}_{t}^{\mathbb{P}}\left(e^{-\gamma\left(\hat{z}_{t}\right)^{\prime} \varepsilon_{t+1}+u_{z}^{\prime} \hat{z}_{t+1}+u_{d} \Delta d_{t+1}}\right) \\
& =-\kappa\left[-\gamma\left(\hat{z}_{t}\right)^{\prime} ; z_{t}\right]+u^{\prime} \mu+u^{\prime} \Phi \hat{z}_{t}+\kappa\left[-\gamma\left(\hat{z}_{t}\right)^{\prime}+u^{\prime} \Sigma\left(\hat{z}_{t}\right) ; z_{t}\right] \\
& =\ln E_{t}^{\mathbb{P}}\left[e^{u_{z}^{\prime} \hat{z}_{t+1}+u_{d} \Delta d_{t+1}}\right]+\kappa\left[-\gamma\left(\hat{z}_{t}\right)^{\prime}+u^{\prime} \Sigma\left(\hat{z}_{t}\right) ; z_{t}\right]-\kappa\left[-\gamma\left(\hat{z}_{t}\right)^{\prime} ; z_{t}\right]-\kappa\left[u^{\prime} \Sigma\left(\hat{z}_{t}\right) ; z_{t}\right]
\end{aligned}
$$

\section{F. Proof of proposition 5}

The exponential-affine solution of the Perron-Frobenius eigenfunction problem can be verified:

$$
\begin{aligned}
\delta e^{u_{z}^{\prime} \hat{z}_{t}} & =E_{t}^{\mathbb{P}}\left[e^{m_{t+1}+u_{z}^{\prime} \hat{z}_{t+1}}\right]=e^{-r_{t}} E_{t}^{\mathbb{Q}}\left[e^{u_{z}^{\prime} \hat{z}_{t+1}}\right] \\
& =e^{-r\left(\hat{z}_{t}\right)-\kappa\left[-\gamma\left(\hat{z}_{t}\right)^{\prime} ; z_{t}\right]+u_{z}^{\prime} A \hat{z}_{t}+\kappa\left[-\gamma\left(\hat{z}_{t}\right)^{\prime}+u_{z}^{\prime} B\left(\hat{z}_{t}\right) ; z_{t}\right]} \\
& =e^{-r(0)-r_{1}(0) \hat{z}_{t}+u_{z}^{\prime} A \hat{z}_{t}+\kappa\left[-\gamma(0)^{\prime}+V(0)^{\prime} ; z\right]-\kappa\left[-\gamma(0)^{\prime} ; z\right]+\kappa_{1}\left[-\gamma(0)^{\prime}+V(0)^{\prime} ; z\right]\left[-\gamma(0)^{\prime}+V(0)^{\prime}\right]+\kappa_{1}\left[\gamma(0)^{\prime} ; z\right] \gamma_{1}(0)+\kappa_{2}[-\gamma(0)+V(0) ; z]-\kappa_{2}[-\gamma(0) ; z]}
\end{aligned}
$$

where we rely on proposition 4 .

\section{G. Additional proofs}

Equivalence of Hansen-Scheinkman and Alvarez-Jermann decompositions. By proposition 3, $u_{z}^{\prime}$ is the equilibrium coefficient of approximate affine real bond yields, i.e., of claims to the cashflow process $d=0$ in equation (20). Accordingly,

$$
\ln (\delta)=\lim _{n \rightarrow \infty}\left[A_{0}^{(n)}-A_{0}^{(n-1)}\right] \quad \ln \left[f\left(x_{t}\right)\right]=B_{0, z}^{(\infty)} \hat{z}_{t}
$$

characterize a solution to the eigenfunction problem, where $\left\{A_{0}^{(n)} ; B_{0, z}^{(n)}\right\}$ are the coefficients in the equilibrium expression of real bond yields. It follows that

$$
m_{t+1}^{T}=\ln (\delta)+B_{0, z}^{(\infty)} \hat{z}_{t}-B_{0, z}^{(\infty)} \hat{z}_{t+1}=-r_{0, t+1}^{(\infty)}
$$

which implies the equivalence. 
Borovicka-Hansen elasticities. To derive the approximate expressions for shock-exposure and shock-price elasticities, define $h_{t+1}(r) \doteq r \alpha_{t} \varepsilon_{t+1}-\kappa\left[r \alpha_{t} ; z_{t}\right]$ and note that, by the law of iterated expectations,

$$
\begin{aligned}
E_{t}^{\mathbb{P}}\left[e^{h_{t+1}(r)} \frac{D_{t+n}}{D_{t}}\right] & =E_{t}^{\mathbb{P}}\left[e^{h_{t+1}(r)+\Delta d_{t+1}} E_{t+1}^{\mathbb{P}}\left(\frac{D_{t+n}}{D_{t+1}}\right)\right]=E_{t}^{\mathbb{P}}\left[e^{h_{t+1}(r)+\Delta d_{t+1}} G_{d, t+1}^{(n-1)}\right] \\
E_{t}^{\mathbb{P}}\left[e^{h_{t+1}(r)} M_{t, t+n} \frac{D_{t+n}}{D_{t}}\right] & =E_{t}^{\mathbb{P}}\left[e^{h_{t+1}(r)+m_{t+1}+\Delta d_{t+1}-n y_{d, t+1}^{(n-1)}}\right]
\end{aligned}
$$

where the term structures of cashflow growth $G_{d, t}^{(n)}$ and yields $y_{d, t}^{(n)}$ have been defined above.

It follows that, under affine approximate term structures:

$$
\begin{aligned}
\varepsilon_{g, t} & =\frac{d}{d r} \ln E_{t}^{\mathbb{P}}\left[e^{h_{t+1}(r)+\Delta d_{t+1}} F_{g, t+1}^{(n-1)}\right]_{r=0}=\frac{d}{d r} \ln E_{t}^{\mathbb{P}}\left[e^{h_{t+1}(r)+\Delta d_{t+1}+A_{g}^{(n-1)}+B_{g}^{(n-1)} \hat{z}_{t+1}}\right]_{r=0} \\
& =\kappa_{1}\left[D\left(\hat{z}_{t}\right)+B_{g}^{(n-1)} B\left(\hat{z}_{t}\right) ; z_{t}\right] \alpha\left(\hat{z}_{t}\right) \\
\varepsilon_{p, t} & =\varepsilon_{g, t}-\frac{d}{d r} \ln E_{t}^{\mathbb{P}}\left[e^{h_{t+1}(r)+m_{t+1}+\Delta d_{t+1}} F_{g, t+1}^{(n-1)}\right]_{r=0}=\varepsilon_{g, t}-\frac{d}{d r} \ln E_{t}^{\mathbb{P}}\left[e^{h_{t+1}(r)+m_{t+1}+\Delta d_{t+1}+A_{d}^{(n-1)}+B_{d, z}^{(n-1)} \hat{z}_{t+1}}\right]_{r=0} \\
& =\kappa_{1}\left[-\gamma\left(\hat{z}_{t}\right)+D\left(\hat{z}_{t}\right)+B_{z}^{(n-1)} B\left(\hat{z}_{t}\right) ; z_{t}\right] \alpha\left(\hat{z}_{t}\right)
\end{aligned}
$$



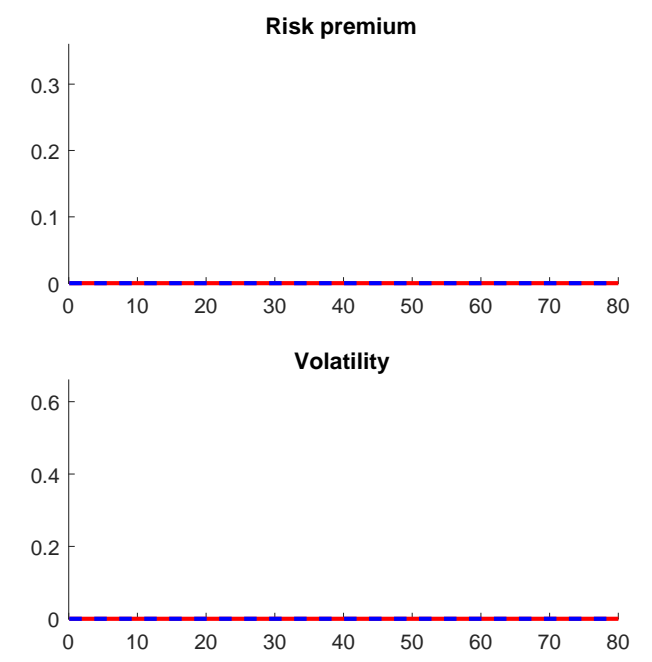

(a) Real bonds.
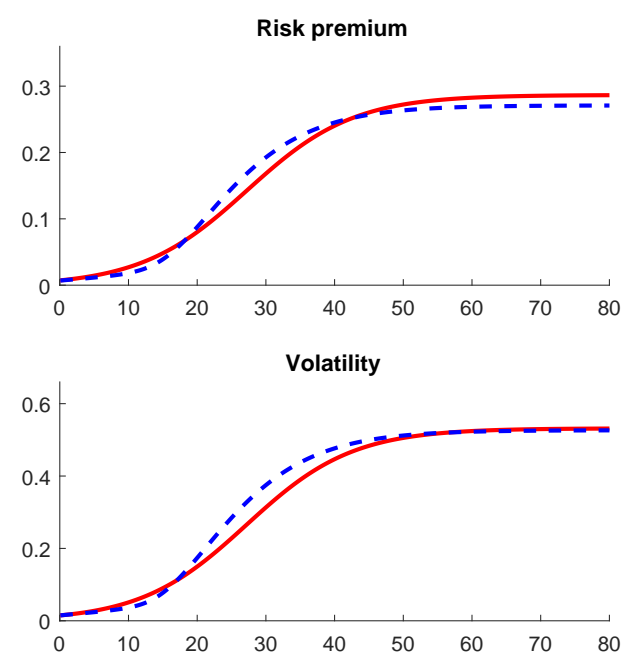

(b) Consumption strips.

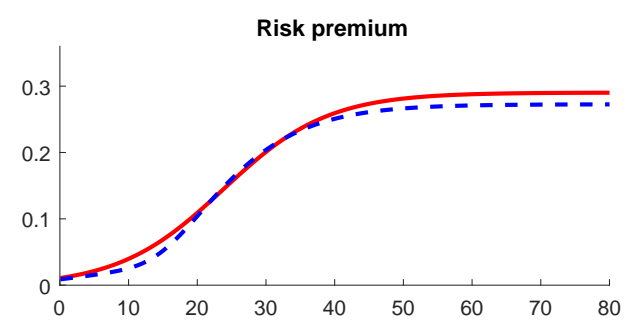

Volatility

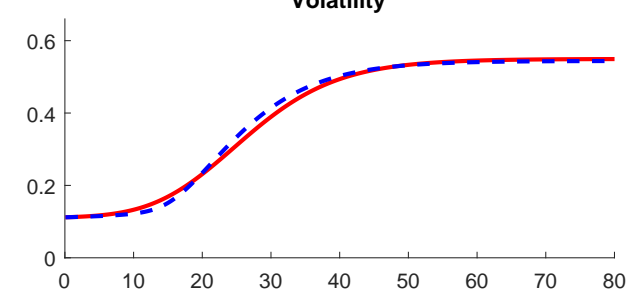

(c) Dividend strips.

Figure 4: Comparison of solution methods to compute average equilibrium term structures of holding-period risk premia $\left\{\ln E_{t} R_{t+1}^{e,(n)}\right\}$ and volatilities $\left\{s t d_{t}\left(r_{t+1}^{(n)}\right)\right\}$ in Campbell and Cochrane (1999). Generalized affine (solid red) and projected solution using cubic splines collocated over 100 Chebyshev nodes and 20-point Gauss-Hermite quadrature (dashed blue). 

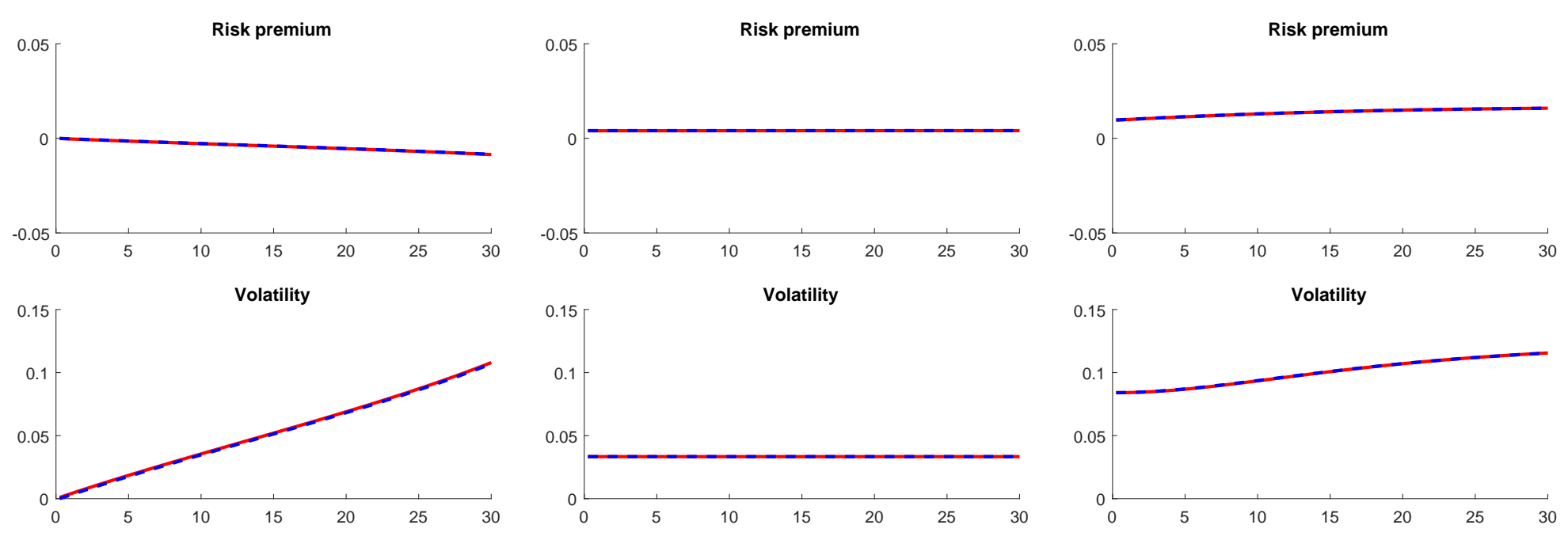

(a) Real bonds.

(b) Consumption strips.

(c) Dividend strips.

Figure 5: Comparison of solution methods to compute average equilibrium term structures of holding-period risk premia $\left\{\ln E_{t} R_{t+1}^{e,(n)}\right\}$ and volatilities $\left\{s t d_{t}\left(r_{t+1}^{(n)}\right)\right\}$ in Wachter (2013). Generalized affine (solid red) and projected solution using Chebyshev polynomials of up to degree eight collocated over a Smolyak grid and 10-point Gauss-Hermite quadrature (dashed blue). 

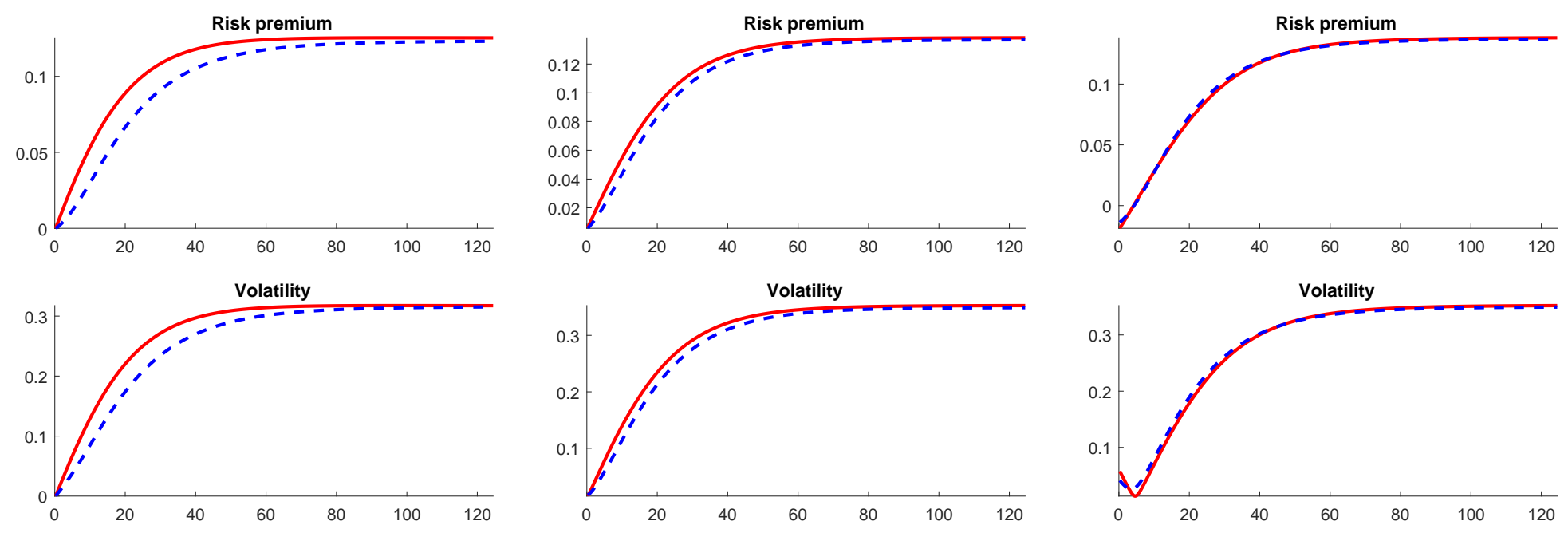

(a) Real bonds.

(b) Consumption strips.

(c) Dividend strips.

Figure 6: Comparison of solution methods to compute average equilibrium term structures of holding-period risk premia $\left\{\ln E_{t} R_{t+1}^{e,(n)}\right\}$ and volatilities $\left\{s t d_{t}\left(r_{t+1}^{(n)}\right)\right\}$ in Jermann (1998)/Chen (2017). Generalized affine (solid red) and projected solution using Chebyshev polynomials of up to degree eight collocated over a Smolyak grid and 10-point Gauss-Hermite quadrature (dashed blue). 


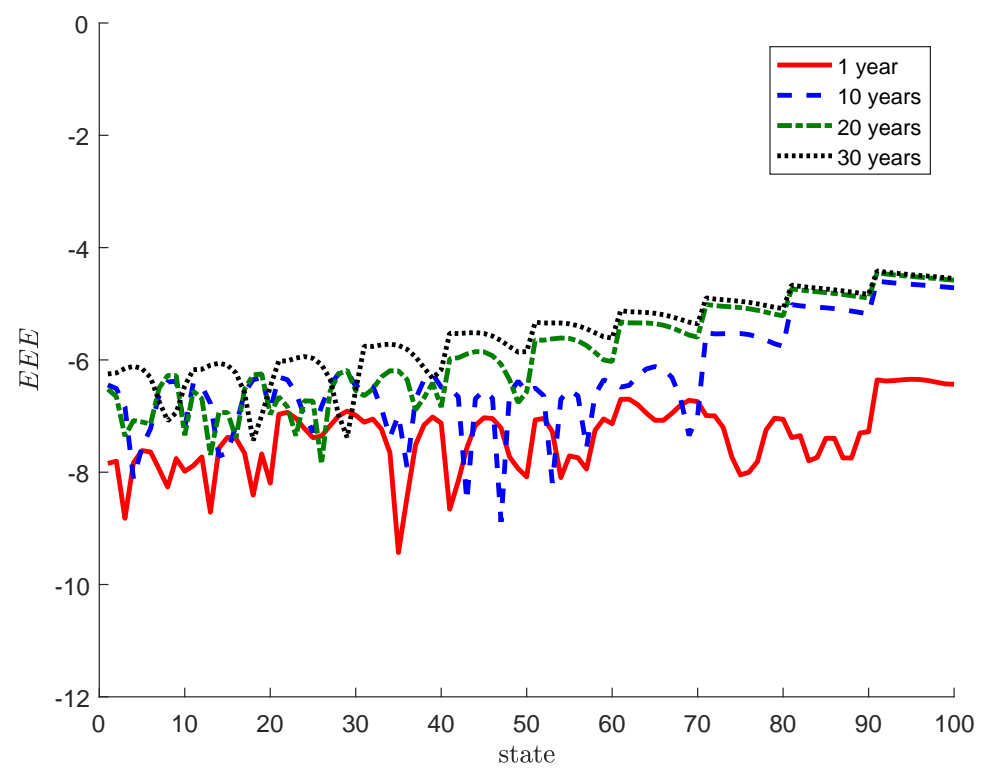

(a) Projected solution for quantities.

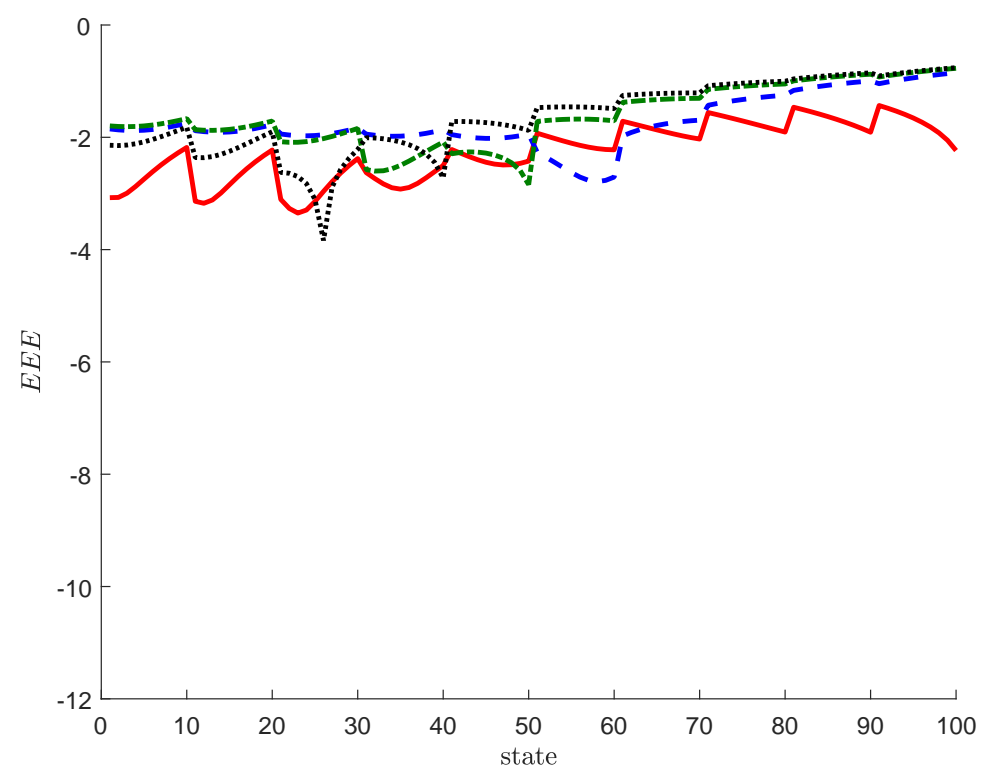

(b) Generalized affine approximation for quantities.

Figure 7: Multiperiod Euler equation errors in Jermann (1998)/Chen (2017). Errors are expressed in $\log _{10}$. Values in the state dimension index different pairs $\left[k a_{t}, \sqrt{1-2 \hat{s}_{t}}\right]$ built as the Cartesian product of 10 equidistant points along each dimension. The projected solution uses Chebyshev polynomials of up to degree eight collocated over a Smolyak grid. Expectations are evaluated using 10-point Gauss-Hermite quadrature. 


\section{References}

F. Alvarez and U. J. Jermann. Using asset prices to measure the cost of business cycles. Journal of Political Economy, 112(6):1223-56, 2004.

F. Alvarez and U. J. Jermann. Using asset prices to measure the persistence of the marginal utility of wealth. Econometrica, 73(6):1977-2016, 2005.

F. Alvarez, A. Atkeson, and P. J. Kehoe. If exchange rates are random walks, then almost everything we say about

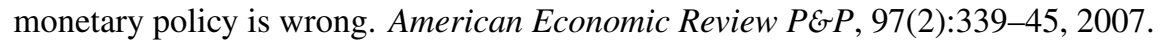

D. Backus, M. Chernov, and S. E. Zin. Sources of entropy in representative agent models. Journal of Finance, 69(1): 51-99, 2014.

D. Backus, A. Ferriere, and S. E. Zin. Risk and ambiguity in models of business cycles. Journal of Monetary Economics, 69:42-63, 2015.

D. Backus, N. Boyarchenko, and M. Chernov. Term structures of asset prices and returns. Journal of Financial Economics, 129:1-23, 2018.

R. Bansal and A. Yaron. Risks for the long run: A potential resolution of asset pricing puzzles. Journal of Finance, 59 (4):1481-1509, 2004.

G. Bekaert, S. Cho, and A. Moreno. New Keynesian macroeconomics and the term structure. Journal of Money, Credit and Banking, 42(1):33-62, 2010.

J. H. v. Binsbergen, J. Fernández-Villaverde, R. S. J. Koijen, and J. F. Rubio-Ramírez. The term structure of interest rates in a DSGE model with recursive preferences. Journal of Monetary Economics, 59:634-48, 2012.

O. J. Blanchard and C. M. Kahn. The solution of linear difference models under rational expectations. Econometrica, 48(5):1305-11, 1980.

N. Bloom. Fluctuations in uncertainty. Journal of Economic Perspectives, 28(2):153-176, 2014.

J. Borovicka and L. P. Hansen. Examining macroeconomic models through the lens of asset pricing. Journal of Econometrics, 183:67-90, 2014.

J. Y. Campbell. Intertemporal asset pricing without consumption data. American Economic Review, 83(3):487-512, 1993.

J. Y. Campbell and J. H. Cochrane. By force of habit: A consumption-based explanation of aggregate stock market behavior. Journal of Political Economy, 107(2):205-51, 1999.

G. Chamberlain and C. A. Wilson. Optimal intertemporal consumption under uncertainty. Review of Economics Dynamics, 3:365-95, 2000.

A. Y. Chen. External habit in a production economy: A model of asset prices and consumption volatility risk. Review of Financial Studies, 30(8):2890-2932, 2017.

A. Y. Chen and F. Palomino. An irrelevance theorem for risk aversion and time-varying risk. Manuscript, 2018.

J. H. Cochrane. Discount rates. Journal of Finance, 66(4):1047-1108, 2011.

N. Coeurdacier, H. Rey, and P. Winant. The risky steady state. American Economic Review PE्GP, 101(3):398-401, 2011.

I. Dew-Becker. Bond pricing with a time-varying price of risk in an estimated medium-scale Bayesian DSGE model. Journal of Money, Credit and Banking, forthcoming, 2014.

J. Fernández-Villaverde, P. Guerrón-Quintana, J. F. Rubio-Ramírez, and M. Uribe. Risk matters: The real effects of volatility shocks. American Economic Review, 101:2530-61, 2011.

J. Fernández-Villaverde, G. Gordon, P. Guerrón-Quintana, and J. F. Rubio-Ramírez. Nonlinear adventures at the zero lower bound. Journal of Economic Dynamics and Control, 57:182-204, 2015a.

J. Fernández-Villaverde, P. Guerrón-Quintana, K. Kuester, and J. F. Rubio-Ramírez. Fiscal volatility shocks and economic activity. American Economic Review, 105:3352-84, 2015 b.

F. Gourio. Disaster risk and business cycles. American Economic Review, 102(6):2734-66, 2012.

L. P. Hansen. Dynamic valuation decomposition within stochastic economies. Econometrica, 80(3):911-67, 2012.

L. P. Hansen and R. Jagannathan. Implications of security market data for models of dynamic economies. Journal of Political Economy, 99(2):225-62, 1991.

L. P. Hansen and J. A. Scheinkman. Long term risk: An operator approach. Econometrica, 77(1):177-234, 2009.

L. P. Hansen and K. J. Singleton. Stochastic consumption, risk aversion, and the temporal behavior of asset returns. Journal of Political Economy, 91(2):249-68, 1983. 
U. J. Jermann. Asset pricing in production economies. Journal of Monetary Economics, 41:257-75, 1998.

H. Jin and K. L. Judd. Perturbation methods for general dynamic stochastic models. Manuscript, 2002.

M. Juillard. Local approximation of DSGE models around the risky steady state. Manuscript, 2011.

G. Kaltenbrunner and L. A. Lochstoer. Long-run risk through consumption smoothing. Review of Financial Studies, 23 (8):3190-3224, 2010.

P. J. Kehoe, P. Lopez, V. Midrigan, and E. Pastorino. Asset prices and unemployment fluctuations. Manuscript, 2018.

J. Kim, S. Kim, E. Schaumburg, and C. A. Sims. Calculating and using second order accurate solutions of discrete time dynamic equilibrium models. Journal of Economic Dynamics and Control, 32:3397-3414, 2008.

P. Klein. Using the generalized Schur form to solve a multivariate linear rational expectations models. Journal of Economic Dynamics and Control, 24:1405-23, 2000.

S. Lang. Real and Functional Analysis, 3rd ed. Springer, New York, 1993.

M. Lettau and H. Uhlig. Can habit formation be reconciled with business cycle facts? Review of Economic Dynamics, 3:79-99, 2000.

P. Lopez. The term structure of the welfare cost of uncertainty. BdF working paper, 521, 2014.

P. Lopez, D. Lopez-Salido, and F. Vazquez-Grande. Nominal rigidities and the term structures of equity and bond returns. FEDS Board of Governors of the Federal Reserve System, 2015-064, 2015.

A. Malkhozov. Asset prices in affine real business cycle models. Journal of Economic Dynamics and Control, 45: 180-193, 2014.

A. Meyer-Gohde. Risk-sensitive linear approximations. Manuscript, 2016.

G. D. Rudebusch and E. T. Swanson. The bond premium in a DSGE model with long-run real and nominal risk. American Economic Journal: Macroeconomics, 4(1):105-143, 2012.

S. Schmitt-Grohé and M. Uribe. Closing small open economy models. Journal of International Economics, 61:163-85, 2003.

S. Schmitt-Grohé and M. Uribe. Solving dynamic general equilibrium models using a second-order approximation to the policy function. Journal of Economic Dynamics and Control, 28:755-75, 2004.

H. Uhlig. Explaining asset prices with external habits and wage rigidities in a DSGE model. American Economic Review PE P, 97(2):239-43, 2007.

A. Verdelhan. A habit-based explanation of the exchange rate risk premium. Journal of Finance, 65(1):123-46, 2010.

J. A. Wachter. A consumption-based model of the term structure of interest rates. Journal of Financial Economics, 79: 365-99, 2006.

J. A. Wachter. Can time-varying risk of rare disasters explain aggregate stock market volatility? Journal of Finance, 68 (3):987-1035, 2013. 